ARTICLE

Received 18 Mar 2016 | Accepted 9 Sep 2016 | Published 18 Oct $2016 \quad$ DOl: 10.1038/ncomms13202 OPEN

\title{
Nicotinic acetylcholine receptor agonist attenuates ILC2-dependent airway hyperreactivity
}

Lauriane Galle-Treger ${ }^{1, \star}$, Yuzo Suzuki ${ }^{1, \star}$, Nisheel Patel ${ }^{1}$, Ishwarya Sankaranarayanan ${ }^{1}$, Jennifer L. Aron ${ }^{1}$, Hadi Maazi ${ }^{1}$, Lin Chen $^{2} \&$ Omid Akbari ${ }^{1}$

Allergic asthma is a complex and chronic inflammatory disorder that is associated with airway hyperreactivity (AHR) and driven by Th2 cytokine secretion. Type 2 innate lymphoid cells (ILC2s) produce large amounts of Th2 cytokines and contribute to the development of AHR. Here, we show that ILC2s express the $\alpha 7$-nicotinic acetylcholine receptor ( $\alpha 7 n A C h R$ ), which is thought to have an anti-inflammatory role in several inflammatory diseases. We show that engagement of a specific agonist with $\alpha 7 n A C h R$ on ILC2s reduces ILC2 effector function and represses ILC2-dependent AHR, while decreasing expression of ILC2 key transcription factor GATA-3 and critical inflammatory modulator NF- $\mathrm{KB}$, and reducing phosphorylation of upstream kinase IKK $\alpha / \beta$. Additionally, the specific $\alpha 7 \mathrm{nAChR}$ agonist reduces cytokine production and AHR in a humanized ILC2 mouse model. Collectively, our data suggest that $\alpha 7 \mathrm{nAChR}$ expressed by ILC2s is a potential therapeutic target for the treatment of ILC2-mediated asthma.

\footnotetext{
${ }^{1}$ Department of Molecular Microbiology and Immunology, Keck School of Medicine, University of Southern California, 1450 Biggy Street NRT 5509 , Los Angeles, California 90033, USA. ${ }^{2}$ Departments of Biological Science and Chemistry, University of Southern California, 1050 Childs Way RIH 201 , Los Angeles, California 90089, USA. * These authors contributed equally to this work. Correspondence and requests for materials should be addressed to O.A. (email: akbari@usc.edu).
} 
A sthma, which is a major worldwide health problem, is a chronic inflammatory disease of the airways with several phenotypes, comprised of both allergic and nonallergic asthma ${ }^{1,2}$. Allergic sensitization in which antigenpresenting cells (APCs) present allergens, followed by T-helper type 2 (Th2) cell skewing and eosinophilic inflammation, are essential for the development of allergic asthma. Obesity, ozone, viral infections, stress and air pollution are associated with nonallergic asthma, the pathogenesis of which involves the innate pathway rather than Th2 cell-mediated immunity ${ }^{3-5}$. Indeed, non-Th2 factors such as interferon- $\gamma$, IL-17 and neutrophils are often found in the lungs of patients with severe non-atopic asthma $^{1,2}$. Moreover, these allergic and non-allergic components may be present in individual patients to various degrees, leading to a complex immune milieu and disease heterogeneity ${ }^{1,2}$.

Innate lymphoid cells (ILCs) are a non-B cell, non-T-cell lymphocyte population in mucosal and lymphoid tissues that are not antigen specific, but respond rapidly to environment factors to induce various types of cytokines ${ }^{6,7}$. Among the ILCs, group 2 ILCs (ILC2s) are directly activated by innate signals from myeloid and epithelial-derived cytokines and alarmins, such as IL-25, IL-33 and proteases, without requiring further differentiation. Following activation, ILC2s produce robust amounts of Th2 cytokines IL-5 and IL-13 to promote eosinophilic inflammation and airway hyperreactivity (AHR); thus, they play an essential role in the pathogenesis of asthma ${ }^{6,7}$. The suggestion that ILC2s are critical for innate immunity activation in asthma is logical as influenza infection ${ }^{5}$ and exposure to proteases and fungi ${ }^{8,9}$ induce AHR by activating innate lymphoid cells. In addition, ILC2s participate in shaping and regulating adaptive immune responses ${ }^{10}$. ILC2-produced IL-5 and IL-13 also contribute to asthma development by respectively recruiting eosinophils in airways and inducing goblet cell mucus production. ILC2s can also directly stimulate a Th2 response in vitro ${ }^{11}$ and facilitate an antigen-specific T-cell response during helminth infection ${ }^{12}$. This evidence suggests that ILC2s are deeply involved in the pathogenesis of asthma by modulating both innate and adaptive immune responses. Thus, regulating the function of ILC2s could be an ideal therapeutic strategy.

The $\alpha 7$ nicotinic acetylcholine receptor (nAChR), mediates rapid excitatory synaptic transmission and is shown to be a potential therapeutic target in neuropsychiatric ${ }^{13}$, neurodegenerative $^{14}$ and inflammatory disease $\mathrm{e}^{15,16}$. In pulmonary allergic inflammation, nicotine, an agonist for nAChR, attenuates Th2 cytokine, IgE and cysteinyl leukotriene levels, resulting in reduced allergic inflammation ${ }^{17,18}$. However, the specific cellular mechanisms by which nAChR activation regulates allergic inflammation are not clearly defined. Given the therapeutic potential of $\alpha 7 \mathrm{nAChR}$ in neurological disorders and inflammatory diseases, a variety of $\alpha 7$-specific ligands have been developed by medicinal chemistry ${ }^{19,20}$ and structural approaches ${ }^{21-23}$. A number of these $\alpha 7 \mathrm{nAChR}$ compounds showed promising efficacy and have advanced to clinical trials ${ }^{19}$. For the initial proof-of-concept studies presented here, we select a leading compound, GTS-21 (also known as DMXBA), that is functionally known to act as an agonist with partial selectivity toward $\alpha 7 \mathrm{nAChR} \mathrm{R}^{24,25}$, and characterize its role in ILC2-dependent AHR. Utilizing this agonist, we demonstrate a critical role for $\alpha 7 \mathrm{nAChR}$ in regulating ILC2-mediated AHR and airway inflammation in a preclinical model of allergic asthma. Moreover, we validate the specificity of the $\alpha 7 \mathrm{nAChR}$ agonist using $\alpha 7 \mathrm{nAChR}$-deficient mice. As expected, the $\alpha 7 \mathrm{nAChR}$ agonist was impaired in suppressing ILC2-dependent AHR in a7nAChR-deficient mice. Reconstitution studies with alymphoid mice suggest that while recipients of wild-type ILC2s responded to suppressive activity of the agonist, recipients of the $\alpha 7 \mathrm{nAChR}$-deficient ILC2s did not show any alteration in AHR or eosinophilia after agonist treatment. These observations establish that $\alpha 7 \mathrm{nAChR}$ expression by ILC2s is crucial for the anti-inflammatory role of the agonist, and our reagent acts specifically through $\alpha 7 \mathrm{nAChR}$. Administration of the $\alpha 7 \mathrm{nAChR}$ agonist inhibits activation of key transcriptional factors, such as GATA-3 and NF- $\kappa$ B. Furthermore, $\alpha 7 \mathrm{nAChR}$ agonist abrogates phosphorylation of $I K K \alpha / \beta$, a critical kinase upstream of the NF- $\kappa \mathrm{B}$ signalling pathway. Using a translational approach, we also show that engagement of $\alpha 7 \mathrm{nAChR}$ results in decreased cytokine production in human ILC2s and a reduction in AHR in a humanized ILC2 model. Our findings provide insight into the regulation of ILC2s that could ultimately be used to generate new therapeutic approaches for ILC2-dependent asthma.

\section{Results}

$\alpha 7 n A C h R$ agonists and $\alpha 7 n A C h R$ expression by ILC2s. Because of the potential therapeutic application in neurological disorders and inflammation, $\alpha 7$-selective ligands have been intensively studied. Li et al. ${ }^{21}$ solved the crystal structure of a receptor chimera constructed from the extracellular domain of $\alpha 7 \mathrm{nAChR}$ and acetylcholine binding protein (AChBP), which represents the closest structural homologue of the native $\alpha 7 \mathrm{nAChR}$. We have also determined structures of $\alpha 7 \mathrm{nAChR}$ bound by agonist and antagonist ${ }^{21,26}$, and performed structure-based screens of $\alpha 7 n$ AChR-specific compounds ${ }^{22}$. Our studies, together with those reported by others, have demonstrated that the crystal structure of the $\alpha 7 \mathrm{nAChR} / \mathrm{AChBP}$ chimera is a much better template for structure-based drug screening than $\mathrm{AChBP}^{23}$, which has been widely used in previous drug screening and design. These studies have identified a variety of potential ligands and allosteric modulators of $\alpha 7 \mathrm{nAChR}$, including many previously known $\alpha 7$-specific compounds. Of these, GTS-21 (DMXB-A) has been previously characterized as a neuronal nAChR ligand. GTS-21 binds to both the $\alpha 4 \beta 2$ and $\alpha 7$ subtypes $^{24,25,27,28}$, but activates only the $\alpha 7$ subtype to a significant extent. In our structure-based drug screen, we selected 4-OH-GTS-21, the active form of GTS-21 in vivo, from the docking analyses. As shown in Fig. 1a, the overall structure is similar to that of $4-\mathrm{OH}-\mathrm{GTS}-21$ bound to $\mathrm{AChBP}^{29}$, including the conformation and orientation of 4-OH-GTS-21 bound to the ligand pocket. On the other hand, our docking analyses reveal that a number of $\alpha 7$-specific residues located at loop C (Arg182 and Glu185) and loop F (Glu158 and Asp160), which are absent in AChBP, interact, or are poised to interact, with functional groups on 4-OH-GTS-21. These observations provide structural bases to guide further synthetic modification of GTS-21 to gain higher selectivity toward the $\alpha 7$ subtype and to modify the functional effects of similar ligands, thus expanding the therapeutic repertoire of agents based on the anabaseine scaffold.

The $\alpha 7 \mathrm{nAChR}$ is present on macrophages ${ }^{15}, \mathrm{~T}$ cells $\mathrm{s}^{30,31}$ and $\mathrm{B}$ cells $^{32}$, and activation of this receptor mediates anti-inflammatory effects. However, there are no studies investigating $\alpha 7 \mathrm{nAChR}$ expression on ILC2s, despite their essential role in the development of asthma. We identified ILC2s as a $\mathrm{Lin}^{-}, \mathrm{CD} 25^{+}, \mathrm{CD} 45^{+}$, $\mathrm{CD} 90.2^{+}$, and $\mathrm{ST}_{2}{ }^{+}$population (Fig. 1b). As mentioned previously, ILC2s are activated by IL-33; however, they are not the only cells that express the IL-33 receptor. As GTS-21 binds to both $\alpha 4$ and $\alpha 7$ subtypes of nAChR, we further assessed mRNA levels of $\alpha 7$ and $\alpha 4 \mathrm{nAChR}$ in various types of immune cells. Interestingly, $\alpha 7 \mathrm{nAChR}$ expression was significantly higher in ILC2s as compared with other ST2 ${ }^{+}$peripheral immune cells (Fig. 1c). Importantly, only $\alpha 7 \mathrm{nAChR}$ in ILC2s, but not $\alpha 4 \mathrm{nAChR}$, was significantly present and up-regulated by in vitro IL-33 treatment (Fig. 1c,d). To confirm this finding at the protein level and also to investigate the effect of IL-25 stimulation, we treated 
a

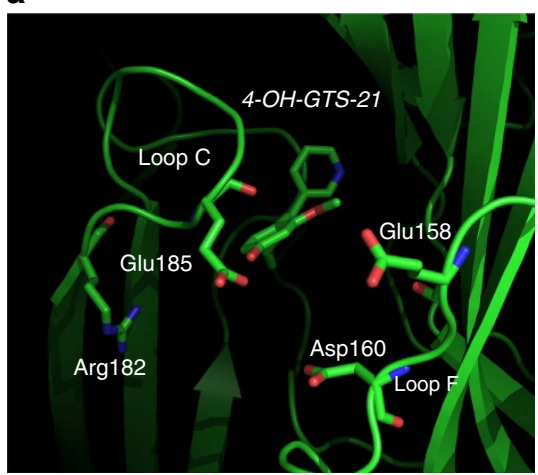

C
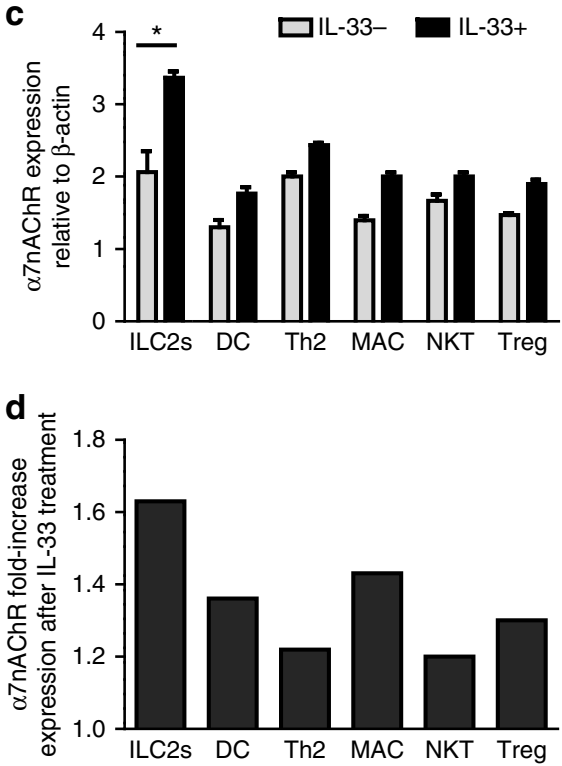

b
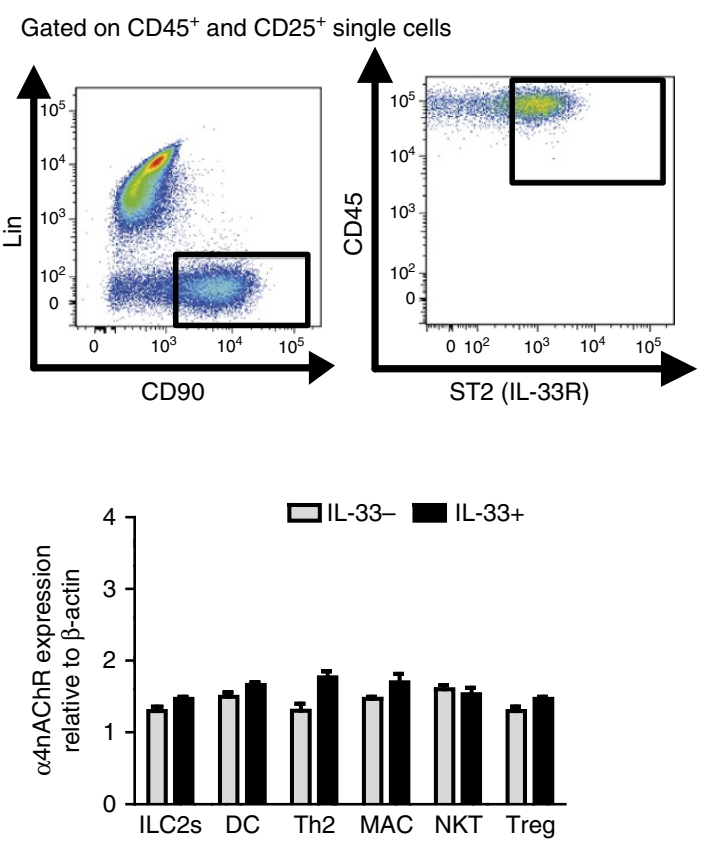

e

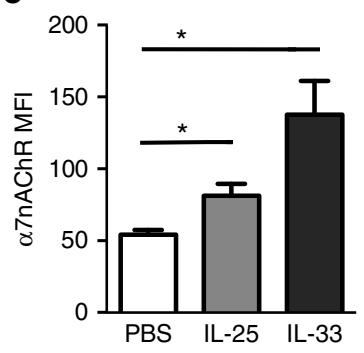

Figure 1 | Structure of $\alpha \mathbf{7 n A C h R}$ agonist and $\alpha \mathbf{7 n A C h R}$ expression on ILC2s. (a) Structure of 4-OH-GTS-21 bound to the $\alpha 7 \mathrm{nAChR}$ chimera. The receptor protein is shown in ribbon; the ligand and nearby protein residues are shown in stick model. The ligand, protein residues and key receptor loop (Loop $\mathrm{C}$ and Loop F) are labeled accordingly. (b) Flow cytometry analysis of lung ILC2s isolated from BALB/cByJ mice, as defined by a lack of lineage markers (CD3e, CD45R, Gr-1, CD11c, CD11b, Ter119, NK1.1, TCR- $\gamma \delta$ and FceRI) and expression of CD90, CD45 and ST2. Dot plots show cells gated on CD45 + and $\mathrm{CD} 25+$ single cells. (c) Peripheral immune cells were sorted from naive BALB/cByJ mice and cultured with rm-IL-2 and rm-IL-7 in the presence or absence of $r m-I L-33$. The expressions of $\alpha 7 n A C h R$ and $\alpha 4 n A C h R$ were quantified at the mRNA levels by quantitative real-time PCR. (d) $\alpha 7 n A C h R$ fold-change expression after IL-33 treatment in peripheral immune cells. (e) Expression of $\alpha 7 n A C h R$ in isolated lung ILC2s from mice intranasally challenged for 3 consecutive days with PBS, IL-25 (5 $\mu \mathrm{g})$, or IL-33 (0.5 $\mu \mathrm{g})$. Data are representative of at least three independent experiments and are presented as means \pm s.e.m. $\left(n=3\right.$; Student's $t$-test, $\left.{ }^{\star} P<0.05\right)$.

mice with intranasal recombinant mouse (rm)-IL-33, (rm)-IL-25, or PBS as a negative control, for three consecutive days. As shown in Fig. 1e, we found, for the first time, that $\alpha 7 \mathrm{nAChR}$ is expressed on ILC2s. Importantly, $\alpha 7 \mathrm{nAChR}$ expression on ILC2s was significantly upregulated in mice treated with IL-25 or IL-33, when compared with the PBS control group. $\alpha 7 \mathrm{nAChR}$ expression was additionally confirmed by cytometry using fluorochromeconjugated $\alpha$-bungarotoxin, a nicotinic cholinergic blocker (Supplementary Fig. 1). Meanwhile, $\alpha 7 \mathrm{nAChR}$ did not alter expression of CD25, CD127 and ST2, known as IL-2R, IL-7R and IL-33R, respectively, and which are essential for development of immune cells (Supplementary Fig. 2).

a7nAChR agonist suppresses cytokine production in ILC2s. Nicotine is known as a major constituent of cigarette smoke, which causes impairment of lung function and exacerbation of asthma. Interestingly, nicotine administration attenuates production of Th2 cytokines and leukotrienes in preclinical models of asthma ${ }^{17}$. Nicotine is an agonist for a variety of pentameric nAChRs made up of different combinations of the sixteen nicotinic receptor subunits, thus it lacks specificity for $\alpha 7 \mathrm{nAChR}^{21,33}$. Therefore, using GTS-21, an agonist specific for $\alpha 7 \mathrm{nAChR}$, we sought to determine whether engagement of $\alpha 7 \mathrm{nAChR}$ would alter ILC2s' function. To address this question quantitatively, pulmonary ILC2s were isolated and cultured with increasing doses $\left(2.5,10\right.$ and $\left.50 \mu \mathrm{g} \mathrm{ml}^{-1}\right)$ of $\alpha 7 \mathrm{nAChR}$ agonist in the presence of rm-IL-33, rm-IL-2 and rm-IL-7. Our results show dose-dependent anti-inflammatory effects of $\alpha 7 \mathrm{nAChR}$ agonist on ILC2s (Fig. 2). These effects were independent of cell viability except at the highest tested dose of the agonist (Fig. 2a,b, right panels). To verify that the actions of the agonist were not due to a reduction in the number of ILC2s, we administered IL-33 to mice in vivo with or without agonist treatment, and then quantified the number of IL-5 ${ }^{+}$and IL- $13^{+}$ILC2s within a determined number of ILC2s (Fig. 2c). Similarly to IL-33, IL-25 
a

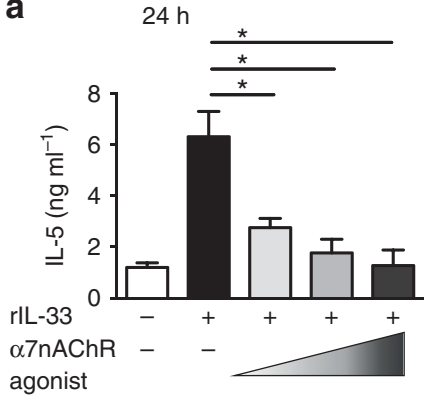

b

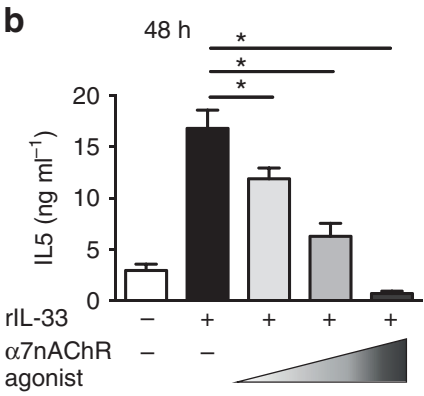

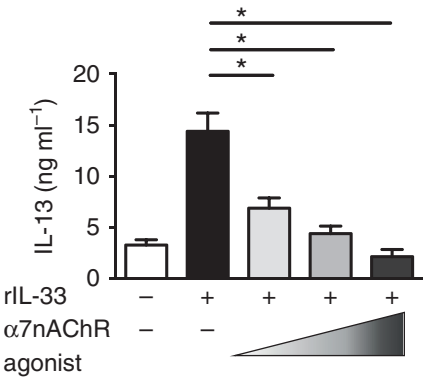

agonist

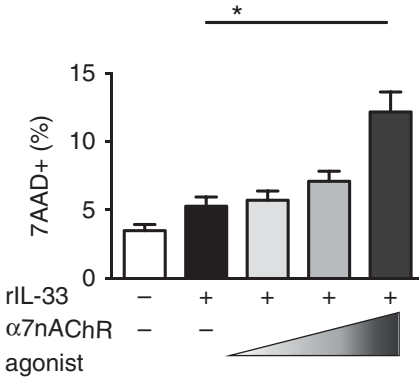

agonist
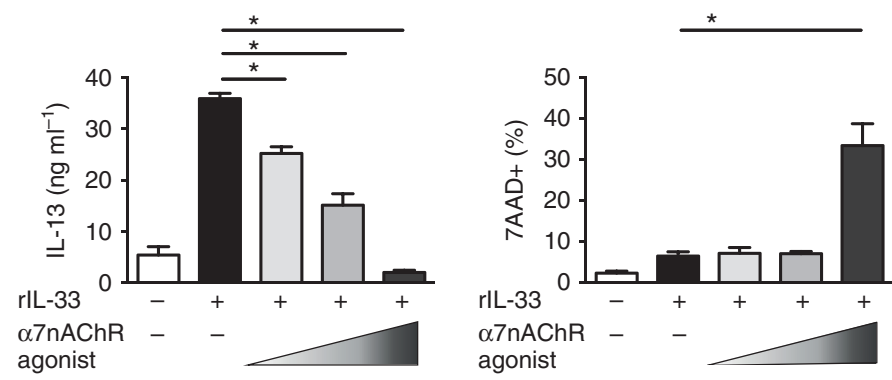

C
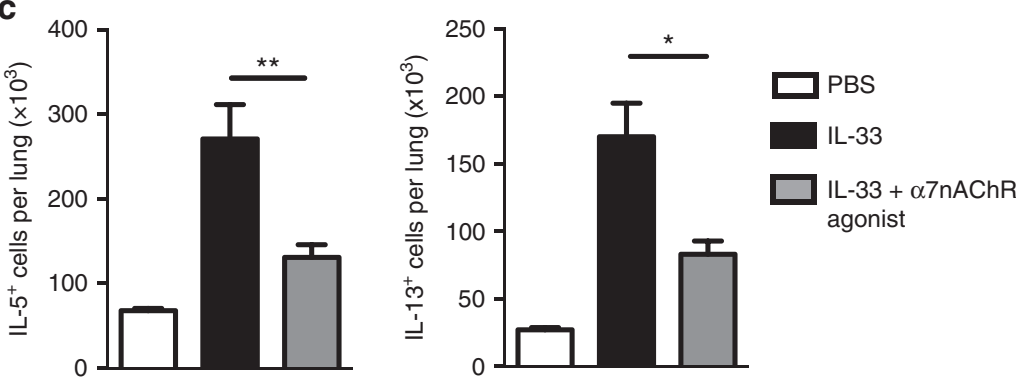

Figure 2 | $\alpha \mathbf{7 n A C h R}$ agonist suppresses IL-5 and IL-13 production from ILC2s in vitro. BALB/cByJ mice were intranasally challenged with recombinant mouse IL-33 on days 1-3. On day 4 lung ILC2s were isolated and re-stimulated with rIL-33, rIL-2 and rIL-7 with or without $\alpha 7 n A C h R$ agonist (2.5, 10 and $50 \mu \mathrm{gl}^{-1}$ ) for $24 \mathrm{~h} \mathrm{(a)} \mathrm{or} 48 \mathrm{~h}(\mathbf{b})$. The levels of IL-5 and IL-13 were measured by ELISA. The viability of cultured ILC2s were assessed by staining with 7-AAD. (c) BALB/CByJ mice were intranasally challenged with recombinant mouse IL-33 on days 1-3, with or without $\alpha 7 n A C h R$ agonist administration. On day 4 lung ILC2s were isolated and the number of IL-5 $5^{+}$and IL-13 ${ }^{+}$cells per $40 \times 10^{4} \mathrm{ILC} 2 \mathrm{~s}$ were quantified. Values are expressed as the mean \pm s.e.m. of five experiments $\left(n=5\right.$; Student's $t$-test, ${ }^{\star} P<0.05$; $\left.{ }^{\star \star} P<0.01\right)$.

increased IL-5 and IL-13 secretion in ILC2s in vitro, though this effect was less robust. IL-25-induced cytokine secretion was also susceptible to suppression by the agonist (Supplementary Fig. 3).

$\alpha 7 n A C h R$ agonist treatment ameliorates ILC2-induced AHR. As reported previously ${ }^{6-9,11,12,34,35}$, the IL-5 and IL-13 cytokines produced by activated ILC2s are essential for eosinophilic inflammation and AHR development. We investigated whether the attenuated ILC2 function by $\alpha 7 \mathrm{nAChR}$ stimulation in vitro could result in inhibition of ILC2-mediated AHR and allergic inflammation. Rag2 deficient mice (devoid of $\mathrm{T}$ and $\mathrm{B}$ cells) were given intranasal (i.n.) rm-IL-33, with or without $\alpha 7 \mathrm{nAChR}$ agonist for three consecutive days (Fig. 3a). As IL-33 administration specifically induces ILC2s, thereby causing AHR, with this model we can readily explore the effect of an $\alpha 7 \mathrm{nAChR}$ agonist in ILC2-mediated AHR. One day after the last challenge, lung function was evaluated by direct measurements of lung resistance $\left(R_{\mathrm{L}}\right)$ and dynamic compliance $\left(C_{\mathrm{dyn}}\right)$, as described in Methods section. We found that stimulating $\alpha 7 \mathrm{nAChR}$ significantly reduced levels of $R_{\mathrm{L}}$ and of $C_{\mathrm{dyn}}$ in response to IL-33, as compared with PBS (Fig. 3b,c). Bronchoalveolar lavage fluid (BALF) analyses also showed decreased eosinophilic infiltration, as well as total cell counts, in $\mathrm{Rag}^{-/-}$mice treated with the $\alpha 7 \mathrm{nAChR}$ agonist (Fig. 3d). Histological analyses revealed that $\alpha 7 \mathrm{nAChR}$ stimulation prevented airway wall thickness and infiltrated cells (Fig. 3e,f). As shown in Fig. 3g, treatment with GTS-21 also significantly suppressed the frequency and absolute number of lung ILC2s. Furthermore, the intracellular cytokine assay also revealed significantly decreased levels of IL-5 and IL-13 producing lung ILC2s in $\alpha 7 \mathrm{nAChR}$ agonist treated mice compared with untreated mice (Fig. 3h). These findings concur with the reduction of AHR and eosinophil counts in BALF (Fig. 3b-d). Thus, $\alpha 7 \mathrm{nAChR}$ agonist represses IL-5 and IL-13 production, eosinophil recruitment, and ILC2-dependent AHR.

$\alpha 7 n A C h R$ on ILC2s is critical for GTS-21 action in vivo. To assess whether the previously observed effects of GTS- 21 are due to its actions on the $\alpha 7 \mathrm{nAChR}$, we challenged WT and $\alpha 7 n A C h R-d e f i c i e n t$ mice with or without rm-IL-33, and with or without $\alpha 7 \mathrm{nAChR}$ agonist, for three consecutive days (Fig. 4a,b). As expected, in the WT mice, the agonist repressed IL-33-induced AHR and eosinophilic infiltration. However, in the absence 
a

i.n. IL-33 $(0.5 \mu \mathrm{g}) \pm \alpha 7 \mathrm{nAChR}$ agonist, or PBS
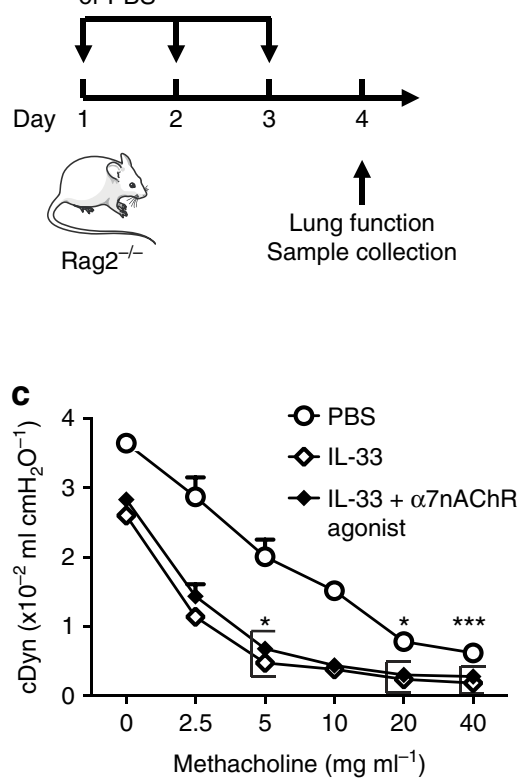

e
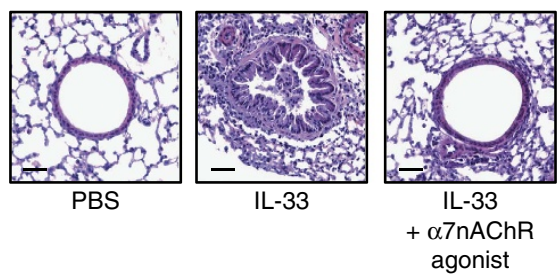

g
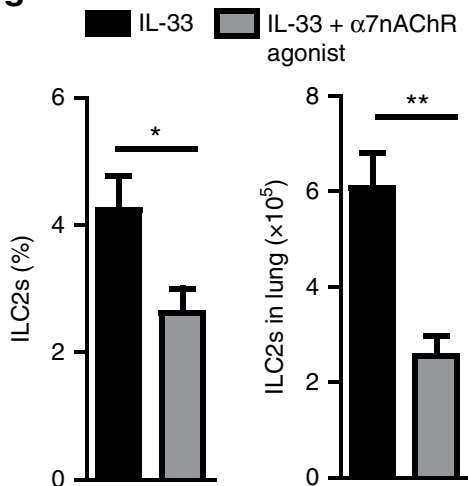

b

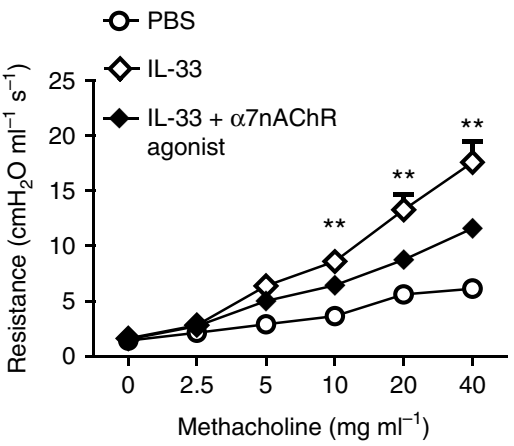

d

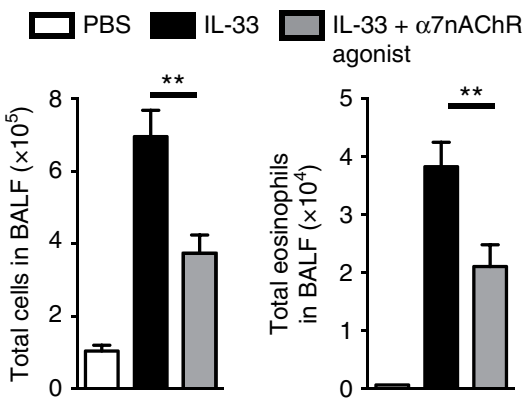

f

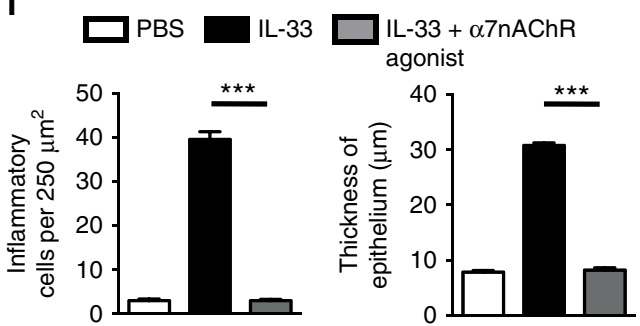

h

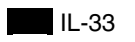

IL-33 $+\alpha 7 n A C h R$ agonist

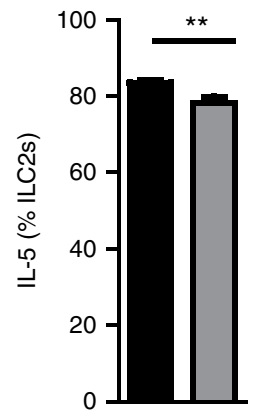

Figure 3 | $\alpha \mathbf{7 n A C h R}$ agonist treatment ameliorates ILC2s-mediated AHR. (a) Rag2-deficient mice were intranasally challenged with recombinant mouse IL-33 or PBS and also received $\alpha 7 n A C h R$ agonist (125 $\mu \mathrm{g}$ ) or PBS on days 1-3 according to $\mathbf{a}$. Measurement of lung function and analyses of bronchoalveolar lavage fluid (BALF) and lung histology followed on day 4. (b,c) Lung resistance and dynamic compliance. (d) Total number of cells and eosinophils in BALF. (e) Hematoxylin and eosin-stained lung sections $(\times 200)$. Scale bars, $50 \mu \mathrm{m}$. (f) Quantification of lung histopathology shown as number of inflammatory cells per $250 \mu \mathrm{m}^{2}$ and thickness of airway epithelium $(\mu \mathrm{m})$. (g) Total number and frequency of lung ILC2s. (h) Percentage of IL-5 ${ }^{+}$and IL-13 ${ }^{+}$lung ILC2s determined by flow cytometry. Data are representative of at least four independent experiments and are presented as means \pm s.e.m. ( $n=8$; Student's $t$-test, ${ }^{\star} P<0.05 ;{ }^{\star \star} P<0.01 ;{ }^{\star \star \star} P<0.001$ ). Mouse outline image provided with permission from Servier Medical Art.

of $\alpha 7 \mathrm{nAChR}$, the agonist affected neither AHR nor eosinophilia. Taken together, these results indicate that engagement of $\alpha 7 \mathrm{nAChR}$ ameliorates ILC2-mediated AHR and allergic inflammation.

Given that a variety of cells participate in allergic inflammation, we wanted to investigate the effect of the agonist specifically on ILC2s. Rag $2^{-/-} \mathrm{GC}^{-/-}$mice lack not only
B and T cells, but NK cells and ILC2s as well. Using methods previously described by our laboratory ${ }^{36}$, we adoptively transferred WT or $\alpha 7 \mathrm{nAChR}$-deficient ILC2s into these Rag2 ${ }^{-/-} \mathrm{GC}^{-/-}$mice, and then treated them with IL-33, with or without $\alpha 7 \mathrm{nAChR}$ agonist. As expected, we observed a decrease of AHR and of eosinophil recruitment in mice injected with WT ILC2s in response to $\alpha 7 \mathrm{nAChR}$ treatment (Fig. $4 \mathrm{c}-\mathrm{e}$ ). 

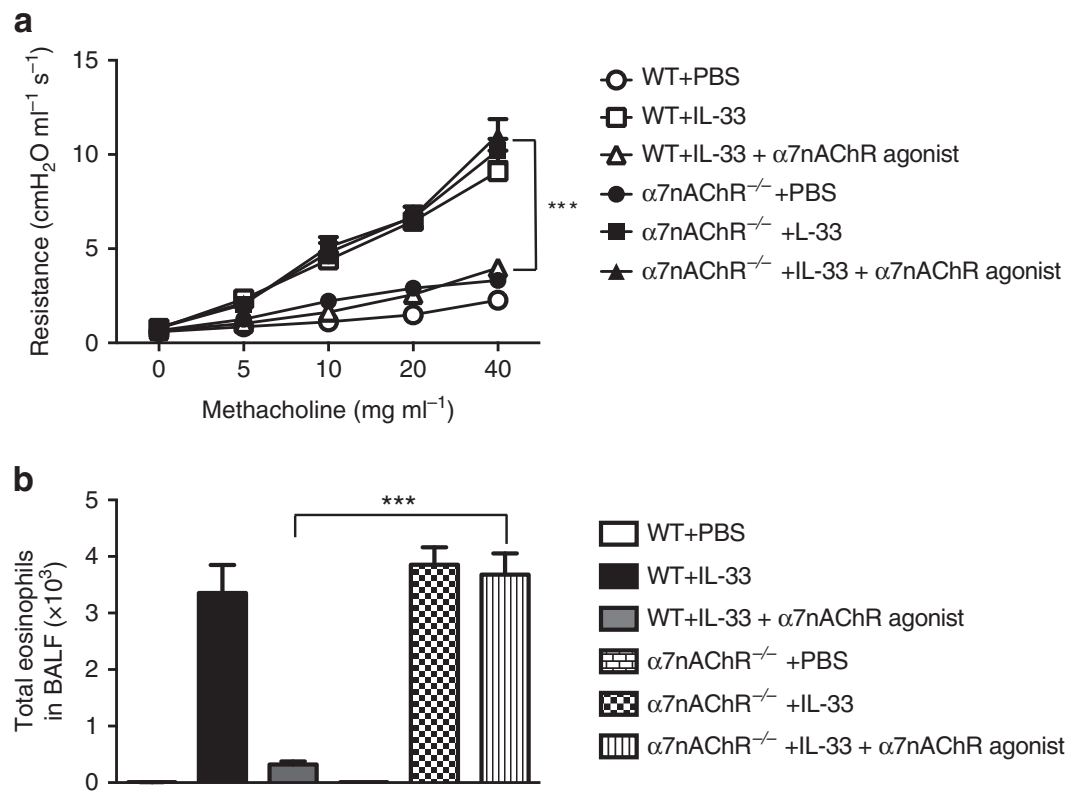

C
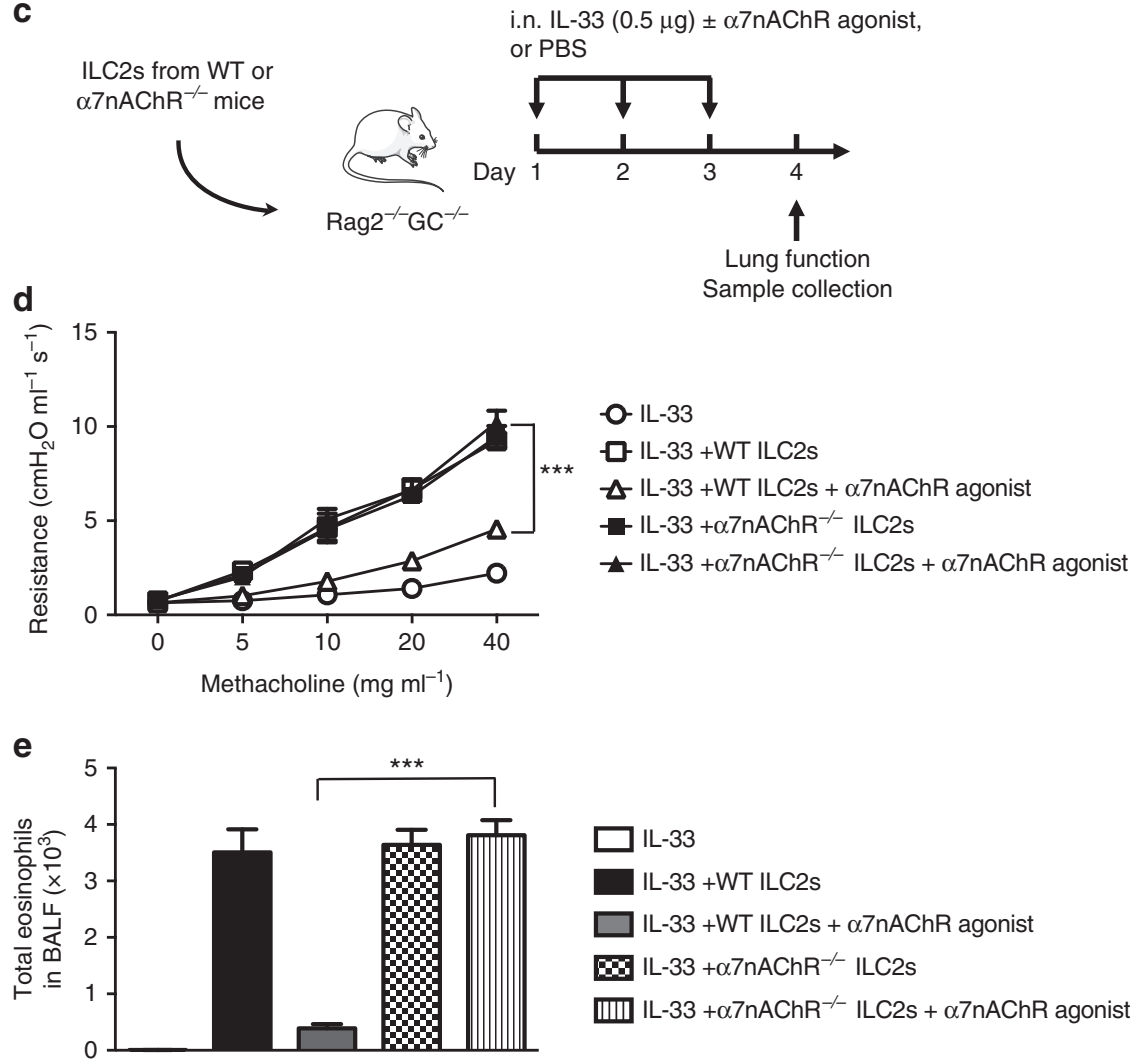

Figure 4 | $\alpha 7 n A C h R$ expression is critical for GTS-21 action on ILC2s in vivo. BALB/cByJ and $\alpha 7 n A C h R^{-/-}$mice were intranasally challenged with rm-IL-33 or PBS, and also given $\alpha 7 n A C h R$ agonist (125 $\mu$ g) or PBS on days 1-3. Measurement of lung function and analysis of BAL followed on day 4 . (a) Lung resistance. (b) Total number of eosinophils in BALF. (c) Rag ${ }^{-/-} \mathrm{GC}^{-/-}$mice were received ILC2s from either WT or $\alpha 7 n A C h R-/-$ mice. After the adoptive transfer, mice were intranasally challenged with rm-IL-33 or PBS; they also received $\alpha 7 \mathrm{nAChR}$ agonist (125 $\mu \mathrm{g})$ or PBS on days 1-3.

Measurement of lung function and analysis of BAL followed on day 4, according to c. (d) Lung resistance. (e) Total number of eosinophils in BALF. Data are representative of at least three independent experiments and are presented as means \pm s.e.m. $\left(n=4\right.$; Student's $t$-test, ${ }^{\star \star \star} P<0.001, \mathrm{WT}$ IL-33 $+\alpha 7 \mathrm{nAChR}$ agonist vs. $\alpha 7 n A C h R-/-I L-33+\alpha 7 n A C h R$ agonist). Mouse outline image provided with permission from Servier Medical Art.

However, in the absence of $\alpha 7 \mathrm{nAChR}$ expression on ILC2s, the agonist affected neither AHR nor eosinophilia. Taken together, these results indicate that engagement of $\alpha 7 \mathrm{nAChR}$ ameliorates ILC2-mediated AHR and allergic inflammation. These results, which are consistent with those seen in WT and $\alpha 7 \mathrm{nAChR}$ deficient mice, demonstrate that the effects we observed are due to ILC2s as opposed to other cells, such as structural cells, which express $\alpha 7 n A C h R$. 
$\alpha 7 n A C h R$ agonist attenuates GATA3 and NF-кB expression. To characterize the mechanism enabling $\alpha 7 \mathrm{nAChR}$ agonist to attenuate ILC2-mediated AHR, we first investigated whether engagement of $\alpha 7 \mathrm{nAChR}$ affects the development or maintenance of pulmonary ILC2s. However, we observed a significantly decreased proliferation rate of pulmonary ILC2s upon $\alpha 7 \mathrm{nAChR}$ agonist treatment by evaluating Ki-67 levels (Fig. 5a). We also evaluated the expression of the transcription factor GATA binding protein-3 (GATA-3), which is essential for the development and maintenance of ILC2 $s^{37-39}$. We found that a7nAChR agonist treatment significantly inhibits GATA-3 transcription in ILC2s (Fig. 5b). This decrease in GATA-3 expression could be involved in the repressed proliferation and function of ILC2s in response to $\alpha 7 \mathrm{nAChR}$ agonist.

Next, to investigate the molecular mechanism of $\alpha 7 \mathrm{nAChR}$ agonist-mediated anti-inflammatory effects, we analysed the gene expression profile of ILC2s with or without $\alpha 7 \mathrm{nAChR}$ agonist treatment by NanoString technology as described in Methods
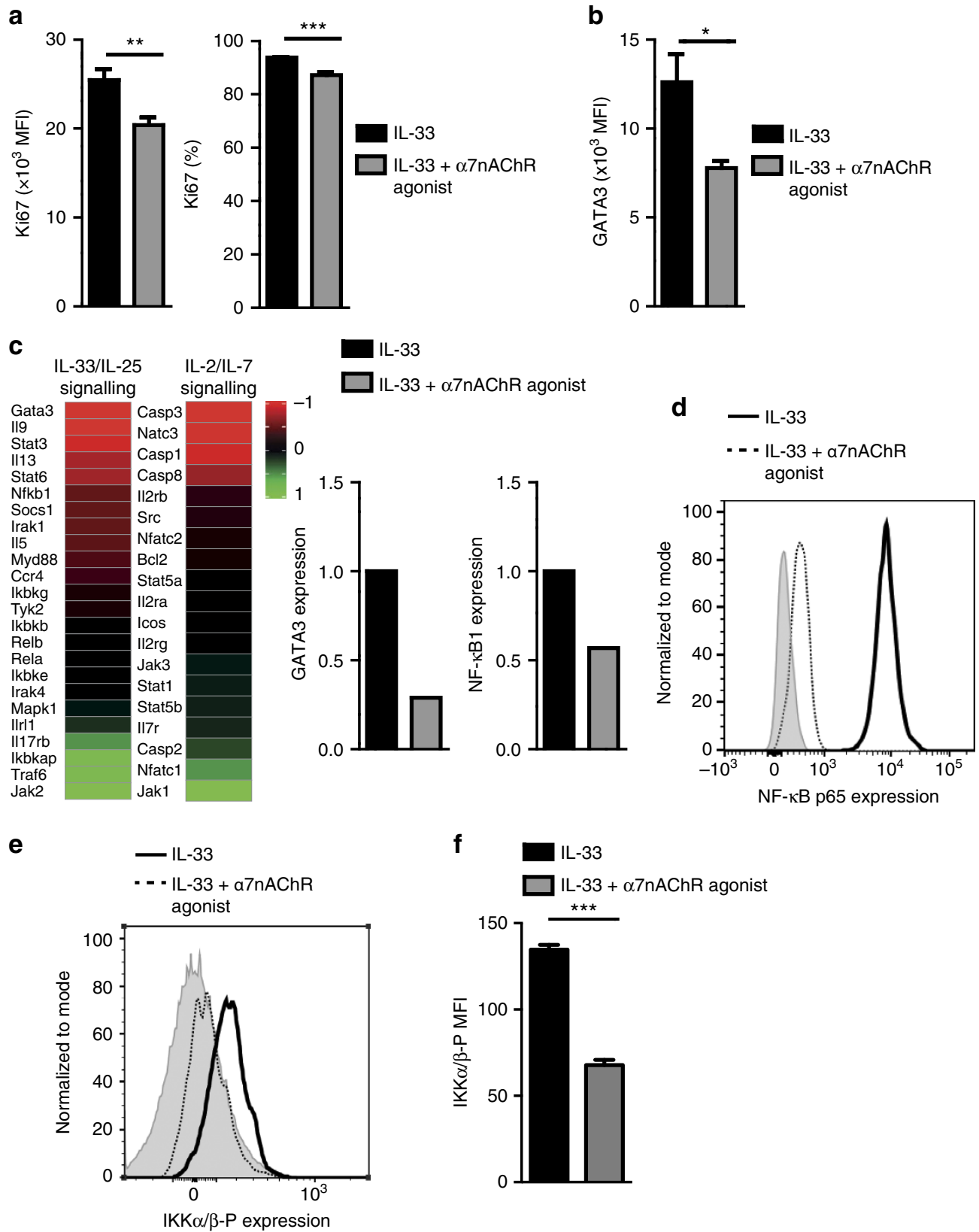

Figure 5 | Cholinergic signal attenuates ILC2 proliferation and GATA3 expression. (a) Mean fluorescence intensity of Ki67 and percentage of Ki67 + cells in isolated lung ILC2s, stimulated with IL-33 in the absence and presence of $\alpha 7 n A C h R$. (b) Mean fluorescence intensity of GATA3 in isolated lung ILC2s, stimulated with IL-33 in the absence and presence of $\alpha 7 \mathrm{nAChR}$. One representative experiment of two is shown $\left(n=5\right.$; Student's $t$-test, ${ }^{\star} P<0.05$; $\left.{ }^{\star \star} P<0.01 ;{ }^{* \star} P<0.001\right)$. (c) Heat plot demonstration of modulation of depicted genes in lung ILC2s treated with $\alpha 7 n A C h R$ agonist. FACS-purified ILC2s from WT mice challenged with rm-IL-33 with or without $\alpha 7 \mathrm{nAChR}$ agonist as described in Fig. 3a were quantified by NanoString nCounter technology. Data range from -1 to +1 for the most reduced and most increased gene expression, respectively. (d) Expression of NF-KB p65 in isolated lung ILC2s from mice challenged with IL-33 with (dotted line) or without (thick line) $\alpha 7 n A C h R$ agonist. The level of isotype-matched stain control is shown as a grey-filled histogram. (e) Expression of phosphorylated IKK $\alpha / \beta$ (Ser176/180) in isolated lung ILC2s stimulated with IL-33, with (dotted line) or without (thick line) $\alpha 7 \mathrm{nAChR}$ agonist for $24 \mathrm{~h}$. The level of isotype-matched stain control is shown as a grey-filled histogram. (f) Mean fluorescence intensity of phosphorylated IKK $\alpha / \beta$ with or without $\alpha 7 n A C h R$ agonist. 
section. Evaluated genes were categorized and displayed in two panels; (1) genes mainly involved in the IL-33/IL-25 signalling pathway, and (2) genes associated with IL-2/IL-7 signalling pathway (Fig. $5 \mathrm{c}$ ). As demonstrated in the IL-33/IL-25 signalling pathway, $\alpha 7 n$ AChR-mediated cholinergic activity inhibited expression of GATA-3 as well as STAT-6, NF- $\kappa$ B, IL-5, IL-9 and IL-13. Meanwhile, STAT-5a, STAT-5b and IL-2 receptors in the IL-2/IL-7 signalling panel were relatively unaffected. We also demonstrated that $\alpha 7 \mathrm{nAChR}$ agonist did not activate factors associated with apoptosis, such as Casp3, Casp8, and Bcl-2. We further confirmed by flow cytometry that $\alpha 7 \mathrm{nAChR}$ agonist reduced NF- $\kappa \mathrm{B}$ p65 expression in ILC2s (Fig. 5d). To better establish the underlying mechanisms, we explored the signalling pathway upstream of NF- $\kappa B$ by measuring the activated form of $I K K \alpha / \beta$, which is phosphorylated on serine residues 176 and 180 . Consistent with our previous results shown in Fig. $5 \mathrm{~d}, \alpha 7 \mathrm{nAChR}$ agonist reduced phosphorylated IKK $\alpha / \beta$ expression in ILC2s (Fig. 5e,f). NF- $\kappa \mathrm{B}$ and STAT-6 are both critical for GATA-3 transcription. The inhibition of GATA-3 expression results in a down-regulation of IL-5, IL-9 and IL-13 secretion from ILC2s. Strikingly, these results demonstrate that $\alpha 7 \mathrm{nAChR}$ stimulation likely reduces maintenance and development of ILC2s by inhibiting GATA-3 transcription, resulting in marked anti-inflammatory effects in the development of ILC2-mediated AHR and allergic inflammation.

$\alpha 7 \mathrm{nAChR}$ agonist treatment attenuates allergen-induced AHR. It has been previously reported that Alternaria alternata can induce $\mathrm{AHR}^{40}$. We further explored the anti-inflammatory effects of $\alpha 7 \mathrm{nAChR}$ agonist in ILC2-mediated AHR with this clinically relevant allergen. Rag2 ${ }^{-/-}$mice were i.n. administered A. alternata extract with or without $\alpha 7 \mathrm{nAChR}$ agonist for 4 consecutive days (Fig. 6a). As expected, $\alpha 7 \mathrm{nAChR}$ agonist treated mice did not develop AHR (Fig. 6b). Accordingly, the number of eosinophils in BALF and lung ILC2s were increased in Alternaria-treated mice. Meanwhile, $\alpha 7 \mathrm{nAChR}$ agonist treatment abolished eosinophils and ILC2s in the lung (Fig. 6c). These results suggest $\alpha 7 \mathrm{nAChR}$ agonist treatment can attenuate ILC2-mediated AHR in response to other allergens besides IL-33.

$\alpha 7 n A C h R$ agonist prevents AHR in humanized ILC2 mice. To assess the effects of $\alpha 7 \mathrm{nAChR}$ agonist in human ILC2s, peripheral blood mononuclear cells (PBMCs) were obtained from healthy donors and ILC2s were sorted on the basis of expression of CD45, CRTH2, CD127 and CD161, and the lack of human lineage markers (CD3, CD14, CD16, CD19, CD20, CD56, CD235a, CD1a and CD123). Sorted ILC2s were cultured with $\alpha 7 \mathrm{nAChR}$ agonist $\left(10 \mu \mathrm{g} \mathrm{ml}^{-1}\right)$ in the presence of recombinant human (rh)-IL-2 $\left(10 \mathrm{ng} \mathrm{ml}^{-1}\right)$, rh-IL-7 $\left(20 \mathrm{ng} \mathrm{ml}^{-1}\right)$ and rh-IL-33 $\left(20 \mathrm{ng} \mathrm{ml}^{-1}\right)$. As observed in our previous experiments (Fig. 2a,b), $\alpha 7 \mathrm{nAChR}$ agonist significantly suppressed human ILC2 activation, by decreasing both IL-5 and IL-13 production (Fig. 7a).

Finally, to confirm the efficacy of $\alpha 7 \mathrm{nAChR}$ agonist treatment in human ILC2-mediated AHR, sorted human ILC2s (hILC2s) were cultured in the presence of rh-IL-2 $\left(20 \mathrm{ng} \mathrm{ml}^{-1}\right)$ and rh-IL-7 $\left(20 \mathrm{ng} \mathrm{ml}^{-1}\right)$ for $48 \mathrm{~h}$. Subsequently, $2.5 \times 10^{5}$ hILC2s were adoptively transferred to Rag2 GC double knockout mice, which lack T, B, NK cells and ILC2s. These humanized mice were given i.n. rh-IL33 (1 $\mu \mathrm{g}$ per mice) with or without $\alpha 7 \mathrm{nAChR}$ agonists $\left(125 \mu \mathrm{g} \mathrm{ml}^{-1}\right)$ for three consecutive days, and then AHR was evaluated (Fig. 7b). Consistent with the murine ILC2 experiments, treatment with $\alpha 7 \mathrm{nAChR}$ agonist dampened ILC2-mediated AHR (Fig. 7c) and allergic inflammation (Fig. $7 \mathrm{~d}, \mathrm{e}$ ) in humanized mice. Thus, $\alpha 7 \mathrm{nAChR}$ mediates an anti-inflammatory signal in both murine and human ILC2s, suggesting $\alpha 7 \mathrm{nAChR}$ activation with an agonist as a novel potential therapeutic strategy for regulating ILC2-mediated lung inflammatory diseases. a

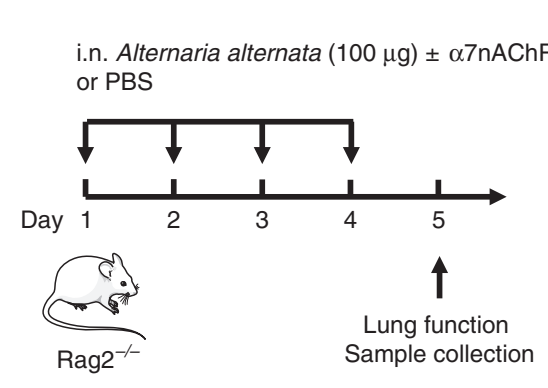

b

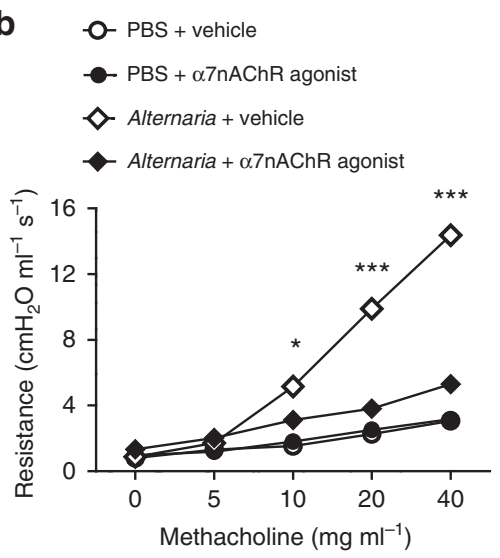

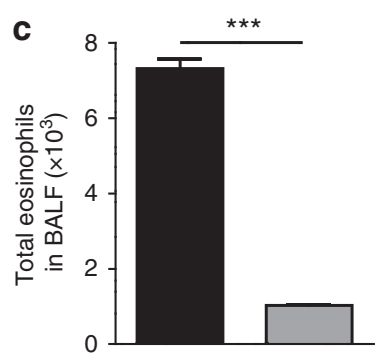

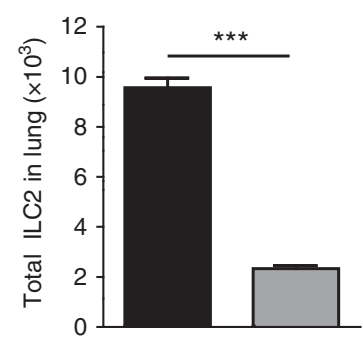

Figure 6 | a7nAChR agonist inhibits Alternaria-induced AHR. (a) Rag2-deficient mice intranasally received an extract of Alternaria alternata with or without $\alpha 7 n A C h R$ agonist on days 1-4 according to a. Measurement of lung function and BALF analysis followed on day 5. (b) Lung resistance. (c) Total number of eosinophils in BALF. Total number of lung ILC2s. Data are representative of at least four independent experiments and are presented as means \pm s.e.m. $\left(n=4\right.$; Student's $t$-test, $\left.{ }^{\star} P<0.05 ;{ }^{\star \star \star} P<0.001\right)$. Mouse outline image provided with permission from Servier Medical Art. 
a $\square$ PBS $\square \mathrm{IL}-33 \quad \square \mathrm{IL}-33+\alpha 7 n A C h R$ agonist
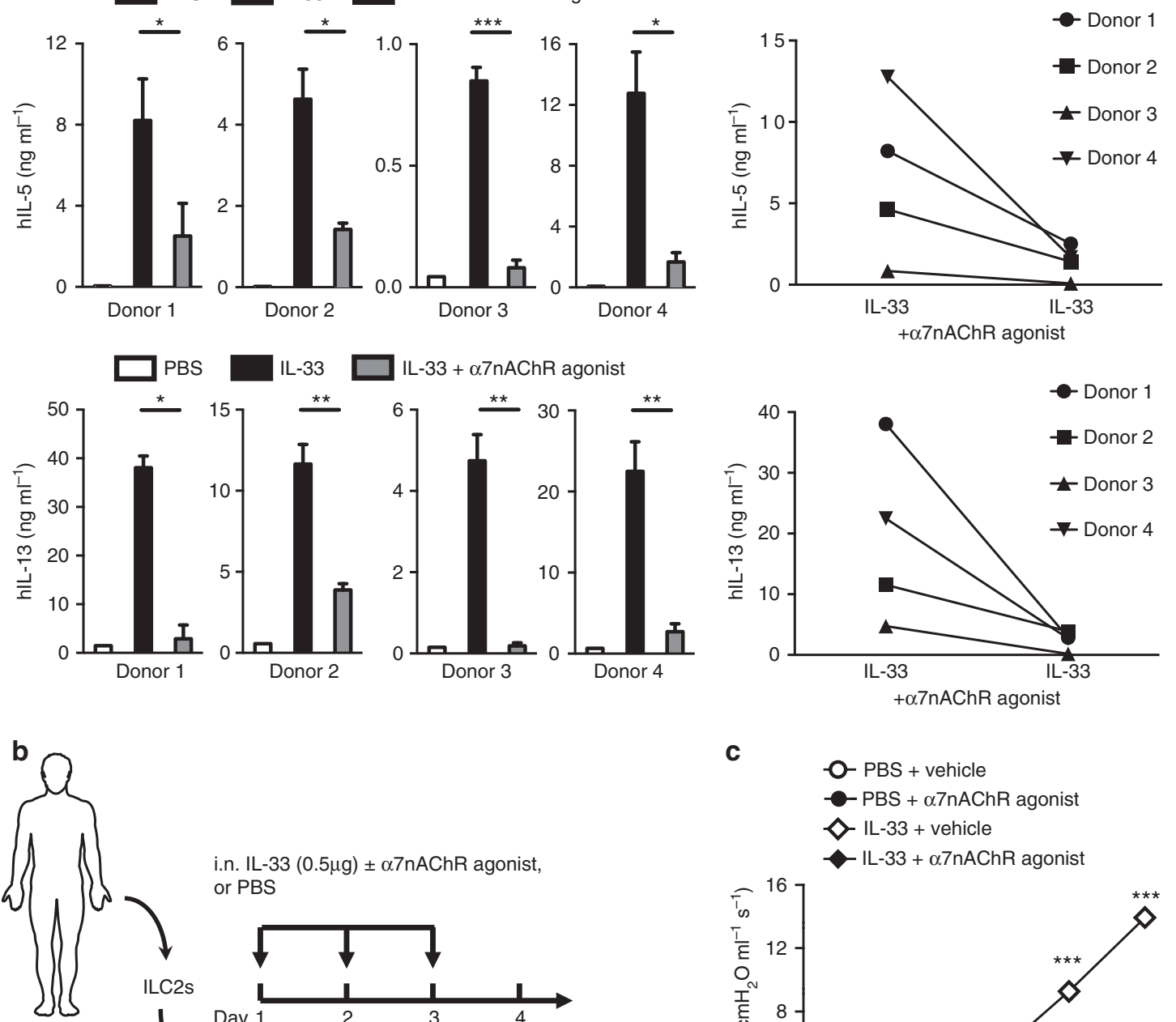

i.n. IL-33 (0.5ug) $\pm \alpha 7 n A C h R$ agonist, or PBS<smiles>CCCCCCC</smiles>

Day 1

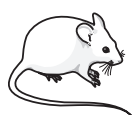

Rag2 $^{-/-} \mathrm{GC}^{-/-}$

ung function

Sample collection

d

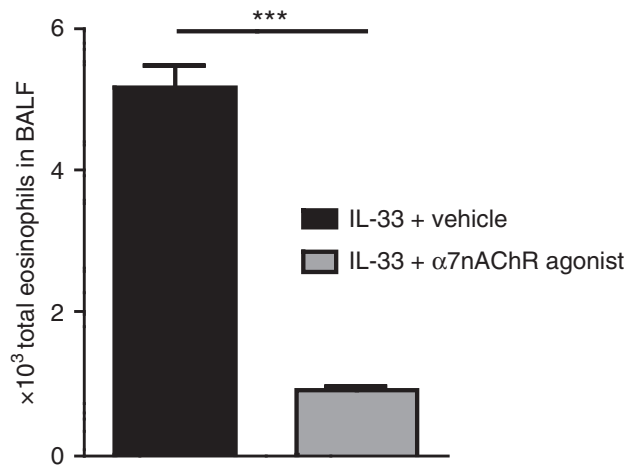

C
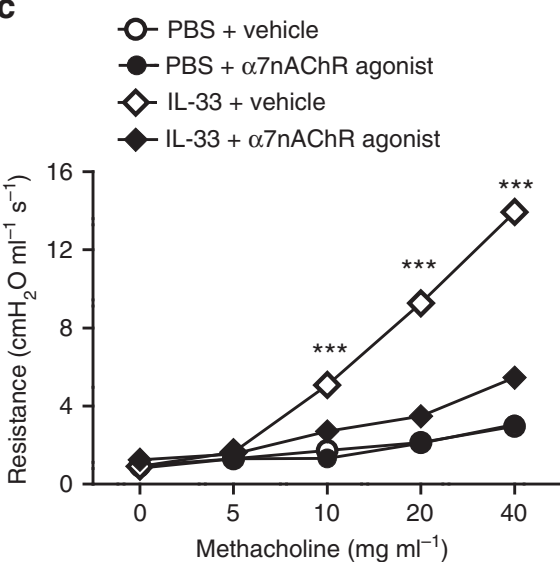

e

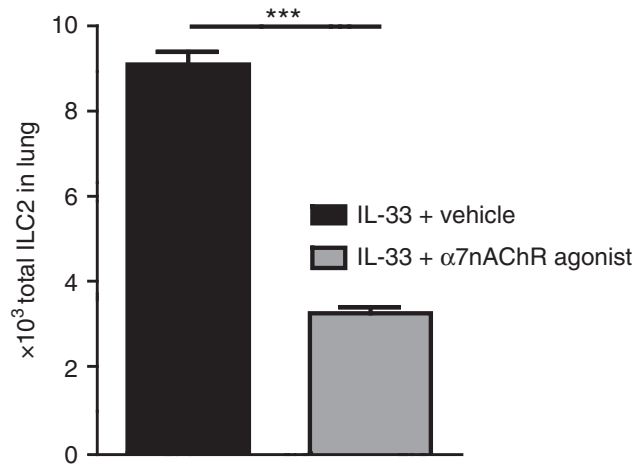

Figure $7 \mid \alpha 7 n A C h R$ agonist prevents human ILC2s mediated AHR and allergic. (a) Human ILC2s were purified from PBMCs via FACS and cultured $\left(10^{4}\right.$ cells per $\left.\mathrm{ml}\right)$ in the presence of recombinant human IL-33 $\left(20 \mathrm{ng} \mathrm{ml}^{-1}\right), \mathrm{IL}-2\left(10 \mathrm{ng} \mathrm{ml}^{-1}\right)$ and IL-7 $\left(20 \mathrm{ng} \mathrm{ml}{ }^{-1}\right)$, with or without $\alpha 7 \mathrm{nAChR}$ agonist for $24 \mathrm{~h}$. In the right panel, data are presented as means of four individual donors. (b) Human peripheral blood mononuclear cells were isolated via FACS and cultured in the presence or absence of rh-IL-2 $\left(10 \mathrm{ng} \mathrm{ml}^{-1}\right)$ and rh-IL-7 $\left(20 \mathrm{ng} \mathrm{ml}^{-1}\right)$ for $48 \mathrm{~h}$, then adoptively transferred into Rag2 ${ }^{-/-} \mathrm{GC}^{-/-}$mice that were intranasally challenged with rm-IL-33 or PBS, with or without $\alpha 7 n A C h R$ agonist $(125 \mu \mathrm{g})$ on days $1-3$. Measurement of lung function and analysis of BALF followed on day 4. (c) Lung resistance. (d) Total number of eosinophils in BALF. (e) Total number of lung ILC2s. Data are representative of at least two independent experiments and are presented as means \pm s.e.m. $\left(n=4\right.$; Student's $t$-test, $\left.{ }^{\star} P<0.05 ;{ }^{\star \star} P<0.01 ;{ }^{\star \star \star} P<0.001\right)$. Mouse and human outline image are provided with permission from Servier Medical Art. 


\section{Discussion}

In the present study, using a leading compound with good selectivity and partial agonist activity towards $\alpha 7 \mathrm{nAChR}$, we examined the involvement of $\alpha 7 \mathrm{nAChR}$ in the pathogenesis of asthma to evaluate its potential as a therapeutic target. We demonstrated, for the first time, that $\alpha 7 \mathrm{nAChR}$ is expressed on ILC2s and upregulated after engagement of the ST2 receptor by IL-33. By inhibiting GATA-3 transcription in ILC2, administration of $\alpha 7 \mathrm{nAChR}$ agonist significantly attenuated the development and function of ILC2s, abolishing both AHR and allergic inflammation. Importantly, we showed this agonist also ameliorated human ILC2-mediated AHR, indicating its potential as a novel therapeutic molecule for asthma patients.

$\alpha 7 \mathrm{nAChR}$ is a neuronal subtype of $\mathrm{nAChR}$ composed of a homopentamer of $\alpha 7$ subunits that mediates pre- and post-synaptic excitation. It is known that $\alpha 7 \mathrm{nAChR}$ is located not only in the brain but also in the periphery, including immune cells such as effector $\mathrm{T}$ cells ${ }^{30,31}, \mathrm{~B}$ cells $^{32}$, Tregs ${ }^{41}$ and macrophages ${ }^{15}$. Using an improved template based on crystal structures, we performed a virtual screen for $\alpha 7 \mathrm{nAChR}$ ligands and selected a leading compound, GTS-21, that has been previously characterized as a functional agonist of $\alpha 7 \mathrm{nAChR}$. Although GTS-21 had previously been shown to have anti-inflammatory effects ${ }^{42,43}$, its role in ILC2-dependent AHR has not been examined to our knowledge. In line with previous studies, we examined a newly identified lymphocyte population of ILC2s and showed that ILC2s expressed $\alpha 7 n A C h R$. Importantly, activated ILC2s upregulated $\alpha 7 \mathrm{nAChR}$ expression more so than other immune cells, indicating that asthma patients having activated ILC2s might benefit greatly from a nicotinic agonist compound such as GTS- 21 .

Nicotine is just one of the over 4,000 chemical constituents in tobacco smoke. Exposure to cigarette smoking causes impaired lung function and increases the risk of developing asthma ${ }^{44,45}$. In asthma, patients who smoke have more symptoms and exacerbations than non-smokers ${ }^{46}$. They also have increased risks of hospitalization and mortality ${ }^{47}$. On the other hand, epidemiological studies have indicated inverse correlations between smoking and incidence of allergic diseases. It is well known that the incidence of hypersensitive pneumonitides such as farmer's lung and bird fancier's lung is lower in the current smoker population than that in non-smokers ${ }^{48-51}$. Furthermore, other clinical studies have observed that asthma incidence could be higher in former smoker populations compared with active smokers ${ }^{44,52}$. In that longitudinal study, the observed increased asthma risks in former smokers were explained by the fact that, in some cases, asthma was self-reported or the supposition that people tend to quit smoking in response to respiratory symptoms. A cohort study also showed that development of allergic sensitization is negatively associated with sustained smoking ${ }^{53}$. In accordance with these clinical findings, nicotine, a major constituent of cigarette smoke and a ligand for $\mathrm{nAChR}$, has shown anti-inflammatory effects in various diseases $31,54,55$. In asthma, nicotine attenuated HDM-induced allergic lung inflammation together with suppression of Th2 cytokines, although the underlying cellular mechanism remains unknown and AHR was not suppressed ${ }^{17}$. Moreover Kearley et al. ${ }^{56}$ recently demonstrated that cigarette smoke has suppressive effects on IL-5 and IL-13 production by ILC2s. In preclinical models of asthma, we clearly demonstrated engagement of $\alpha 7 \mathrm{nAChR}$ opposes the development of AHR and allergic inflammation through an ILC2-mediated mechanism. The differences in outcome likely resulted from the experimental design; our group administered a specific compound for $\alpha 7 \mathrm{nAChR}$ together with rm-IL-33 or A. alternata to mice, whilst Sopori et al. examined nicotine itself and HDM-induced
$\mathrm{AHR}^{21,33}$. To establish that our previous observations were dependent of $\alpha 7 \mathrm{nAChR}$ expression on ILC2s, we demonstrated that the $\alpha 7 \mathrm{nAChR}$ agonist had no effect on IL-33-challenged mice that were deficient for $\alpha 7 \mathrm{nAChR}$ on ILC2s. Collectively, our data can explain the cellular mechanisms behind the clinical anti-inflammatory observations.

We demonstrated that $\alpha 7 \mathrm{nAChR}$ activation altered ILC2 function in response to both exogenous IL-33 and A. alternata. Administration of either IL-33 and IL-25 activates ILC2s to induce AHR independently of the adaptive immune system ${ }^{5,6,57}$, and IL-33 was reported to be more potent than IL-25 in this regard $^{57}$. A. alternata exposure also activates ILC2s and causes steroid-resistant AHR associated with elevated IL-33 in vivo ${ }^{40}$. ILC2s rapidly produce huge amounts of IL5 and IL-13 in response to stimuli. A recent study showed that ILC2-derived IL-13 is capable of inducing differentiation among Th2 cells ${ }^{35}$. Based on these reports, regulating ILC2s could potentially control allergic inflammation arising from not only the innate, but also adaptive, immune pathway. By targeting ILC2 function, $\alpha 7$ nAChR agonist treatments could prove remarkably therapeutic for various allergic diseases.

To explore the mechanisms underlying the anti-inflammatory effects of the $\alpha 7 \mathrm{nAChR}$ agonist, we initially demonstrated the engagement of $\alpha 7 \mathrm{nAChR}$ caused reduced number of ILC2s in the lung, suggesting involvement of the cholinergic signal in cell fate and maintenance. We next found that $\alpha 7 \mathrm{nAChR}$ agonist significantly suppressed Ki67 expression, a cellular marker for proliferation in ILC2s. In contrast, expression of anti-apoptotic factor Bcl-2 on ILC2s was unaffected by $\alpha 7 \mathrm{nAChR}$ stimulation. In accordance with these in vivo data, viability of ILC2s in culture and results from the NanoString assay were comparable. These results suggest that cholinergic signal transmission regulates proliferation, but not cell death, in ILC2s. Our findings are consistent with the cholinergic underpinnings of anti-inflammatory mechanisms in other diseases of inflammation, such as autoimmune arthritis and experimental autoimmune encephalomyelitis $31,54,55$.

On one hand, Dowling et al. ${ }^{58}$ also described that nicotine could inhibit the NF- $\kappa \mathrm{B}$ pathway in an $\alpha 7 \mathrm{nAChR}$-dependent manner. We demonstrated by cytometry that in response to the agonist, the expression of the activated NF- $\kappa \mathrm{B}$ p 65 subunit was reduced. Similarly we also observed a decrease in the expression at the mRNA level of NF- $\mathrm{BB} 1$. However, with the Nanostring Technology we also noticed that RelA expression was unaffected. This discrepancy could be explained by the fact that $\mathrm{NF}-\kappa \mathrm{B}$ is mainly regulated at the post-translational level by phosphorylation. Phosphorylation of the NF- $\kappa B$ p65 subunit on certain residues plays a key role in regulating $\mathrm{NF}-\kappa \mathrm{B}$ activation and function. We also validate our results by assessing $I K K \alpha / \beta$, upstream of $N F-\kappa B$ and observed a significant reduction in $\mathrm{IKK} \alpha / \beta$ after agonist treatment.

On the other hand, in cancer immunity, nicotine administration enhances tumour growth by promoting cell proliferation and suppressing apoptosis ${ }^{59-62}$. Strikingly, these results suggest that cholinergic signal is involved in the pathogenesis of various diseases, with a distinct role in each disease.

In addition to attenuated proliferation, $\alpha 7 \mathrm{nAChR}$ agonist also inhibited GATA-3 expression in ILC2s. GATA-3, a double zinc-finger transcription factor, is required for the development of Th2 cells and important for the production of IL- 5 and IL-13 (refs 63-65). Recently, GATA-3 was also reported to be essential for differentiation and maintenance of ILC2s, and their production of IL-5 and IL-13 (refs 37-39). Moreover, higher expression of GATA-3 is associated with an increase in ILC2-derived IL-13 (ref. 66). Collectively, these results indicate an underlying mechanism for the anti-inflammatory role of 
$\alpha 7 n A C h R$ agonist in asthma: modulating GATA-3 expression and proliferation in ILC2s, which subsequently attenuates Th2 cytokine production from ILC2s, preventing the development of AHR and allergic inflammation.

We further investigated whether this cholinergic antiinflammatory effect was relevant in a humanized mice model, in which human ILC2s were adoptively transferred to $\mathrm{Rag}^{-/-}$ IL2 $\mathrm{rg}^{-1-}$ mice and administered IL-33 to induce AHR. This unique system allows one to directly evaluate human ILC2-mediated $\mathrm{AHR}^{36}$. In accordance with the effects seen in murine ILC2s, $\alpha 7 \mathrm{nAChR}$ agonist significantly suppressed human ILC2 function and dampened human ILC2-mediated AHR. Taken in their entirety, our results suggest that enhancing cholinergic signal might be an effective therapeutic strategy for patients with ILC2-mediated asthma.

In conclusion, this is the first report to reveal that the engagement of $\alpha 7 \mathrm{nAChR}$ on ILC2s suppresses AHR. Therefore, our results suggest a protective role of cholinergic signalling in the pathogenesis of asthma, and present $\alpha 7 \mathrm{nAChR}$ agonists as novel therapeutic candidates for controlling ILC2-mediated inflammatory lung diseases.

\section{Methods}

Mice. Female BALB/cByJ, RAG2 deficient (C.B6(Cg)-Rag2 ${ }^{\text {tm1.1Cgn } / J) ~ m i c e, ~}$ RAG2 GC deficient (C; 129S4-Rag2 ${ }^{\text {tm1.1Flv }}$ IL2rg tm1.1Flv /J) mice and $\alpha 7 \mathrm{nAChR}$ deficient (B6.129S7-Chrnat ${ }^{\text {tmlBay/J) }}$ (6-8 weeks old) were purchased from Jackson Laboratory (Bar Harbor, ME). Rag2-deficient and Rag2 GC-deficient mice were bred in our facility at the Keck School of Medicine, University of Southern California (USC). Animal studies were approved by the USC Institutional Animal Care and Use Committee and conducted in accordance with the USC Department of Animal Resources' guidelines. All human studies were approved by USC institutional review board and conducted according to the principles of the Declaration of Helsinki. Participants gave written informed consent to before their inclusion in the study, and were identified by number.

Crystallization and structure determination of agonist. The structure of the agonist 4-OH-GTS-21 was docked into the ligand site of the crystal structure of the $\alpha 7 \mathrm{nAChR}$ chimera ${ }^{21}$ using our previously published computation docking procedures $^{22}$. The figure is made by PyMOL (The PyMOL Molecular Graphics System, v 1.8, Schrodinger, LLC).

Measurement of airway hyperreactivity. Mice were i.n. administered carrier-free recombinant mouse IL-33 (BioLegend, San Diego, CA, $0.5 \mu \mathrm{g}$ per mouse in $50 \mu \mathrm{l}$ ) with or without $125 \mu \mathrm{l}$ of $\alpha 7 \mathrm{nAChR}$ agonist (kindly provided by Lin Chen) on 3 consecutive days. Mice were i.n. administered carrier-free recombinant mouse IL-25 (BioLegend, San Diego, CA, $5 \mu \mathrm{g}$ per mouse in $50 \mu \mathrm{l}$ ) with or without $125 \mu \mathrm{l}$ of $\alpha 7 \mathrm{nAChR}$ agonist on three consecutive days. For Alternaria alternata experiments, mice were i.n. administered A. alternata (Greer Labs, Lenoir, NC, $100 \mu \mathrm{g}$ per mouse in $50 \mu \mathrm{l}$ ) with or without $125 \mu \mathrm{g} \alpha 7 \mathrm{nAChR}$ agonist for 4 consecutive days. One day after the last challenge, mice were anesthetized using i.p. injection of ketamine $\left(10 \mathrm{mg} \mathrm{ml}^{-1}\right)$ and xylazine $\left(1 \mathrm{mg} \mathrm{ml}^{-1}\right)$. Measurements of airway resistance and dynamic compliance were conducted with the Fine Pointe RC System (Buxco Research Systems, Wilmington, NC), in which mice were mechanically ventilated using a modified version as previously described ${ }^{36,67}$. Mice were sequentially challenged with aerosolized PBS (baseline), followed by increasing doses of methacholine. Maximum lung resistance $\left(R_{\mathrm{L}}\right)$ and minimum compliance $\left(C_{\mathrm{dyn}}\right)$ values were recorded during a 3 -min period after each methacholine challenge.

Collection of BAL cells and lung histology. After measurements of AHR, the trachea was canulated and the lungs lavaged three times with $1 \mathrm{ml}$ ice cold PBS to collect BALF cells as previously described ${ }^{68}$. BALF cells were stained with allophycocyanin (APC)-labeled anti-Ly-6G/Ly-6C (clone RB6-8C5, BioLegend, San Diego, CA), Alexa Fluor-labeled anti-CD19 (clone 6D5, BioLegend), phycoerythrin (PE)-labeled anti-Singlec-F (clone E50-2440, BD Pharmingen, San Diego, CA), and PE-Cy (PE-Cy7) labeled anti-CD45 (clone 30-F11, BioLegend), peridinin-chlorophyll-protein complex-Cy5.5 (PerCP-Cy5.5) labeled anti-CD3e (clone145-2C11, eBioscience, San Diego), and eFluor-450 labeled anti-CD11b (clone M1/70, eBioscience), and APC-Cy7 labeled anti-CD11c (clone N418, BioLegend). Transcardinal perfusion of the lungs with cold PBS was then performed to remove red blood cells and the lungs fixed and harvested for histology with $4 \%$ paraformaldehyde in PBS. After fixation, the lungs were embedded in paraffin, cut into $4 \mu \mathrm{m}$ sections, and stained with hematoxylin and eosin (H\&E).
Flow cytometry. Biotinylated anti-mouse lineage (CD3e (145-2C11), CD45R (RA3-3B2), Gr-1 (RB6-8C5), CD11c (N418), CD11b (M1/70), Ter119 (TER-119), NK1.1 (PK136), TCR- $\beta$ (H57-597), TCR- $\gamma \delta$ (GL3), and FceRI $\alpha$ (MAR-1), Streptavidin-FITC, FITC anti-mouse CD3 (145-2C11), BV510 anti-mouse CD4 (RM4-5), APC-Cy7 anti-mouse CD25 (PC61), PE anti-mouse CD25 (PC61), BV421 anti-mouse CD25 (PC61), BV510 anti-mouse CD90.2 (53-2.1), APC anti-mouse CD127 (A7R34), PE-Cy7 anti-mouse CD127 (A7R34), FITC antimouse CD45 (30F-11), PE/Cy7 anti-mouse CD45 (30-F11), PE anti-mouse IL-5 (TRFK5), BV421 anti-mouse GATA3 (16E10A23), 7-AAD Viability staining solution, purchased from BioLegend (San Diego, CA). PE anti-mouse ST2 (IL-33R, RMST2-2), PerCP-eFluor710 anti-Mouse ST2 (RMST2-2), Streptavidin APC-eFluor780, Alexa Fluor647 labeled anti-IL-4 (clone 11B11), eFluor 450 anti-mouse CD45 (30F-11), eFluor450 anti-CD44 (clone IM7), eFluor-660 anti-mouse Ki-67 (SollA15), PE/Cy7 anti-mouse IL-13 (eBio13A), anti-mouse BCL-2 (10C4), were purchased from eBioscience. Alexa Fluor 488 anti-mouse p65-NF- $\mathrm{B}$ (532301) was purchased from R\&D Systems (dilution 1:50). Alexa Fluor 647 conjugated $\alpha$-bungarotoxin (Invitrogen, San Diego, CA). PE anti-mouse Phospho-IKK $\alpha / \beta$ (Ser 176/180) (16A6) was purchased from Cell Signaling Technology (dilution 1:50). PE anti-rat $\alpha 7 \mathrm{nAChR}$ (319) was purchased from Santa Cruz Biotechnology (dilution 1:50). This antibody has been used to identify this receptor previously in several studies ${ }^{69}$. Lineage marker antibodies were used at the dilution of 1:400, unless mentioned otherwise other antibodies were used at the dilution of 1:200

BD Cytofix Fixation Buffer and BD Phosflow Perm Buffer III were purchased from BD Biosciences (San Jose, CA). Flow cytometry was carried out on the FACSCanto II and FACSARIA III (BD Biosciences) and the data were analysed with FlowJo version 8.6 software (TreeStar, Ashland, Oregon).

Identification of mouse ILC2s. Lung ILC2s were defined as lack of classical lineage markers (CD3e, CD45R, Gr-1, CD11c, CD11b, Ter119, NK1.1, TCR- $\beta$, TCR- $\gamma \delta$ and FceRI $\alpha$ ) and $\mathrm{CD} 25^{+}, \mathrm{CD} 45^{+}, \mathrm{CD} 90.2^{+}$, and $\mathrm{ST} 2{ }^{+}$populations.

Intracellular staining. Intracellular staining was performed using BD Cytofix/ Cytoperm kit (BD Bioscience, San Jose, CA) according to the manufacturer's instructions. For analysis of GATA3 and Ki-67 expression, freshly isolated cells were fixed and permeabilized using Fixation/Permeabilization buffer kit (eBioscience) according to the manufacturer's instructions and as previously described ${ }^{70}$.

Humanized mice and purification of human ILC2. For human peripheral ILC2s, peripheral blood mononuclear cells (PBMCs) were first isolated from human fresh blood by diluting the blood 1:1 in PBS, adding to SepMate-50 separation tubes (STEMCELL Technologies Inc, Vancouver, Canada) prefilled with 15-ml Lymphoprep each (Axis-Shield, Oslo, Norway), and centrifuging at 1,200g for $15 \mathrm{~min}$. Human PBMCs were then washed in PBS and stained with antibodies against human lineage markers (CD3, CD14, CD16, CD19, CD20, CD56, CD235a, CD1a, CD123), CRTH2, CD161, CD127 and CD45. Thereafter, human ILC2s were defined as $\mathrm{CD} 45+$ lineage- $\mathrm{CRTH} 2+\mathrm{CD} 127+\mathrm{CD} 161+$ and purified by flow cytometry using BD FACS ARIA III (BD Biosciences, San Jose, CA) with a purity of $>95 \%$. Purified human ILC2s were cultured with rh-IL2 $\left(20 \mathrm{ng} \mathrm{ml}^{-1}\right)$ and rh-IL-7 $\left(20 \mathrm{ng} \mathrm{ml}^{-1}\right)$ for $48 \mathrm{~h}$, then adoptively transferred to Rag2 GC double knockout mice $\left(2.5 \times 10^{5}\right.$ cells per mouse) followed by i.n. administration of recombinant human IL-33 ( $0.5 \mu \mathrm{g}$ per mouse) with or without $\alpha 7 \mathrm{nAChR}$ agonist on days 1-3. On day 4 , lung function was measured and BAL was performed and analysed.

In vitro stimulation of ILC2s. Murine ILC2s were isolated from BALB/cByJ mice to $>95 \%$ purity using the FACSARIA III cell sorter. Isolated murine ILC2s $\left(5.0 \times 10^{4}\right.$ per ml) were stimulated with rm-IL-33 $\left(20 \mathrm{ng} \mathrm{ml}^{-1}\right)$, rm-IL-2 $\left(10 \mathrm{ng} \mathrm{ml}^{-1}\right)$ and $\mathrm{rm}-\mathrm{IL}-7\left(10 \mathrm{ng} \mathrm{ml}^{-1}\right)$ in the presence or absence of increasing doses of $\alpha 7 \mathrm{nAChR}$ agonist $\left(2.5 \mu \mathrm{g} \mathrm{ml} l^{-1}, 10 \mu \mathrm{g} \mathrm{ml}^{-1}\right.$ and $\left.50 \mu \mathrm{g} \mathrm{ml}^{-1}\right)$ for 1 or 2 days. Isolated murine ILC2s $\left(5.0 \times 10^{4}\right.$ per ml) were stimulated with $\mathrm{rm}$-IL-25 $\left(10 \mathrm{ng} \mathrm{ml}^{-1}\right)$ in the presence or absence of $\alpha 7 \mathrm{nAChR}$ agonist $\left(10 \mu \mathrm{g} \mathrm{ml}^{-1}\right)$ for $24 \mathrm{~h}$. For human ILC2s in vitro culture, isolated human ILC2s $\left(1.0 \times 10^{5} \mathrm{ml}^{-1}\right)$ were cultured with rh-IL-33 $\left(20 \mathrm{ng} \mathrm{ml}^{-1}\right)$, rh-IL-2 $\left(10 \mathrm{ng} \mathrm{ml}^{-1}\right)$, and rh-IL-7 $\left(20 \mathrm{ng} \mathrm{ml}^{-1}\right)$ in the presence of $\alpha 7 \mathrm{nAChR}\left(10 \mu \mathrm{g} \mathrm{ml}^{-1}\right)$. Levels of cytokines were measured by ELISA (eBioscience), according to the manufacturer's instructions.

Reverse transcription-PCR. The expression of $\alpha 7$ and $\alpha 4$ genes was quantitated at the mRNA levels by quantitative real-time PCR (qPCR). Total RNA was extracted with the RNeasy Mini Kit (Qiagen, Valencia, CA), and $\alpha 7 \mathrm{nChR}$ (forward, $5^{\prime}$ CTCTGACTGTCTTCATGCTGCT- ${ }^{\prime}$ and reverse, $5^{\prime}$-ATCATGGTGCTGGC GAAG- $3^{\prime}$ ) and $\alpha 4 \mathrm{nAChR}$ (forward, $5^{\prime}$-CGTCCAGTACATTGCAGACC- $3^{\prime}$ and reverse, $5^{\prime}$-ATGACCATGGCCACGTATTT- $3^{\prime}$ ) genes were quantified at the mRNA level by quantitative real-time PCR (qPCR). Total RNA was extracted with the RNeasy Mini Kit (Qiagen, Valencia, CA), and the $\alpha 7$ and $\alpha 4$ mRNA expressions were measured using the TaqMan gene expression assay at the Applied Biosystems 7500 system (Applied Biosystems, Carlsbad, CA) in accordance with the manufacturer's protocol. 
Gene expression analysis with NanoString nCounter technology. The difference in the abundance of scripts between ILC2s purified from rm-IL33 administered mice with or without $\alpha 7 \mathrm{nAChR}$ agonist were analysed with NanoString nCounter technology. Heat plots were generated with R statistical software.

Statistical analysis. A student $t$-test was used for comparisons between each group. $P$ values of $<0.05$ were considered significant. All data are expressed as the mean \pm s.e.m.

Illustrations. In Figs 3,4,6 and 7 and thumbnail picture we used vector elements from Servier Medical Art PowerPoints under a Creative Commons License.

Data availability. The authors declare that the data supporting the findings of this study are available within the article and its Supplementary Information files.

\section{References}

1. Kim, H. Y., DeKruyff, R. H. \& Umetsu, D. T. The many paths to asthma: phenotype shaped by innate and adaptive immunity. Nat. Immunol. 11, 577-584 (2010).

2. Lambrecht, B. N. \& Hammad, H. The airway epithelium in asthma. Nat. Med. 18, 684-692 (2012).

3. Johnston, R. A. et al. Allergic airway responses in obese mice. Am. J. Respir. Crit. Care Med. 176, 650-658 (2007).

4. Kim, E. Y. et al. Persistent activation of an innate immune response translates respiratory viral infection into chronic lung disease. Nat. Med. 14, 633-640 (2008).

5. Chang, Y. J. et al. Innate lymphoid cells mediate influenza-induced airway hyper-reactivity independently of adaptive immunity. Nat. Immunol. 12, 631-638 (2011)

6. Yu, S., Kim, H. Y., Chang, Y. J., DeKruyff, R. H. \& Umetsu, D. T. Innate lymphoid cells and asthma. J. Allergy Clin. Immunol. 133, 943-950 (2014).

7. Artis, D. \& Spits, H. The biology of innate lymphoid cells. Nature 517, 293-301 (2015)

8. Bartemes, K. R. et al. IL-33-responsive lineage- CD25 + CD44(hi) lymphoid cells mediate innate type 2 immunity and allergic inflammation in the lungs. J. Immunol. 188, 1503-1513 (2012).

9. Halim, T. Y., Krauss, R. H., Sun, A. C. \& Takei, F. Lung natural helper cells are a critical source of Th2 cell-type cytokines in protease allergen-induced airway inflammation. Immunity 36, 451-463 (2012).

10. Molofsky, A. B. et al. Interleukin-33 and interferon-gamma counter-regulate group 2 innate lymphoid cell activation during immune perturbation. Immunity 43, 161-174 (2015).

11. Mirchandani, A. S. et al. Type 2 innate lymphoid cells drive CD4 + Th2 cell responses. J. Immunol. 192, 2442-2448 (2014).

12. Neill, D. R. et al. Nuocytes represent a new innate effector leukocyte that mediates type-2 immunity. Nature 464, 1367-1370 (2010).

13. Martin, L. F., Kem, W. R. \& Freedman, R. Alpha-7 nicotinic receptor agonists: potential new candidates for the treatment of schizophrenia. Psychopharmacology 174, 54-64 (2004).

14. D'Andrea, M. R. \& Nagele, R. G. Targeting the alpha 7 nicotinic acetylcholine receptor to reduce amyloid accumulation in Alzheimer's disease pyramidal neurons. Curr. Pharm. Des. 12, 677-684 (2006).

15. Wang, H. et al. Nicotinic acetylcholine receptor alpha7 subunit is an essential regulator of inflammation. Nature 421, 384-388 (2003).

16. Borovikova, L. V. et al. Vagus nerve stimulation attenuates the systemic inflammatory response to endotoxin. Nature 405, 458-462 (2000).

17. Mishra, N. C. et al. Nicotine primarily suppresses lung Th2 but not goblet cell and muscle cell responses to allergens. J. Immunol. 180, 7655-7663 (2008).

18. Mishra, N. C. et al. Nicotine inhibits Fc epsilon RI-induced cysteinyl leukotrienes and cytokine production without affecting mast cell degranulation through alpha 7/alpha 9/alpha 10-nicotinic receptors. J. Immunol. 185, 588-596 (2010)

19. Hurst, R., Rollema, H. \& Bertrand, D. Nicotinic acetylcholine receptors: from basic science to therapeutics. Pharmacol. Ther. 137, 22-54 (2013).

20. Mazurov, A. A., Speake, J. D. \& Yohannes, D. Discovery and development of alpha7 nicotinic acetylcholine receptor modulators. J. Med. Chem. 54, 7943-7961 (2011).

21. Li, S. X. et al. Ligand-binding domain of an alpha7-nicotinic receptor chimera and its complex with agonist. Nat. Neurosci. 14, 1253-1259 (2011).

22. Dey, R. \& Chen, L. In search of allosteric modulators of a7-nAChR by solvent density guided virtual screening. J. Biomol. Struct. Dyn. 28, 695-715 (2011).

23. Akdemir, A. et al. Identification of novel alpha7 nicotinic receptor ligands by in silico screening against the crystal structure of a chimeric alpha7 receptor ligand binding domain. Bioorg. Med. Chem. 20, 5992-6002 (2012).
24. van Haaren, F., Anderson, K. G., Haworth, S. C. \& Kem, W. R. GTS-21, a mixed nicotinic receptor agonist/antagonist, does not affect the nicotine cue. Pharmacol. Biochem. Behav. 64, 439-444 (1999).

25. Kem, W. R. et al. Hydroxy metabolites of the Alzheimer's drug candidate 3-[(2,4-dimethoxy)benzylidene]-anabaseine dihydrochloride (GTS-21): their molecular properties, interactions with brain nicotinic receptors, and brain penetration. Mol. Pharmacol. 65, 56-67 (2004).

26. Huang, S. et al. Complex between alpha-bungarotoxin and an alpha7 nicotinic receptor ligand-binding domain chimaera. Biochem. J. 454, 303-310 (2013).

27. Stevens, K. E. et al. Continuous administration of a selective alpha7 nicotinic partial agonist, DMXBA, improves sensory inhibition without causing tachyphylaxis or receptor upregulation in DBA/2 mice. Brain Res. 1352, 140-146 (2010).

28. Kem, W. R. Alzheimer's drug design based upon an invertebrate toxin (anabaseine) which is a potent nicotinic receptor agonist. Invert. Neurosci. 3, 251-259 (1997).

29. Hibbs, R. E. et al. Structural determinants for interaction of partial agonists with acetylcholine binding protein and neuronal alpha7 nicotinic acetylcholine receptor. EMBO J. 28, 3040-3051 (2009).

30. Nizri, E., Hamra-Amitay, Y., Sicsic, C., Lavon, I. \& Brenner, T. Anti-inflammatory properties of cholinergic up-regulation: a new role for acetylcholinesterase inhibitors. Neuropharmacology 50, 540-547 (2006).

31. Nizri, E. et al. Activation of the cholinergic anti-inflammatory system by nicotine attenuates neuroinflammation via suppression of Th1 and Th17 responses. J. Immunol. 183, 6681-6688 (2009).

32. Skok, M. V., Grailhe, R., Agenes, F. \& Changeux, J. P. The role of nicotinic receptors in B-lymphocyte development and activation. Life Sci. 80, 2334-2336 (2007).

33. Dellisanti, C. D., Yao, Y., Stroud, J. C., Wang, Z. Z. \& Chen, L. Crystal structure of the extracellular domain of $\mathrm{nAChR}$ alphal bound to alpha-bungarotoxin at 1.94A resolution. Nat Neurosci. 10, 953-962 (2007).

34. Walker, J. A., Barlow, J. L. \& McKenzie, A. N. Innate lymphoid cells--how did we miss them? Nat. Rev. Immunol. 13, 75-87 (2013).

35. Halim, T. Y. et al. Group 2 innate lymphoid cells are critical for the initiation of adaptive $\mathrm{T}$ helper 2 cell-mediated allergic lung inflammation. Immunity $\mathbf{4 0}$, 425-435 (2014).

36. Maazi, H. et al. ICOS:ICOS-ligand interaction is required for type 2 innate lymphoid cell function, homeostasis, and induction of airway hyperreactivity. Immunity 42, 538-551 (2015).

37. Hoyler, T. et al. The transcription factor GATA-3 controls cell fate and maintenance of type 2 innate lymphoid cells. Immunity 37, 634-648 (2012).

38. Klein Wolterink, R. G. et al. Essential, dose-dependent role for the transcription factor Gata3 in the development of IL-5 + and IL-13 + type 2 innate lymphoid cells. Proc. Natl Acad. Sci. USA 110, 10240-10245 (2013).

39. Mjosberg, J. et al. The transcription factor GATA3 is essential for the function of human type 2 innate lymphoid cells. Immunity 37, 649-659 (2012).

40. Castanhinha, S. et al. Pediatric severe asthma with fungal sensitization is mediated by steroid-resistant IL-33. J. Allergy Clin. Immunol. 136, 312-22.e7 (2015).

41. Wang, D. W. et al. Stimulation of alpha7 nicotinic acetylcholine receptor by nicotine increases suppressive capacity of naturally occurring CD4 + CD25 + regulatory T cells in mice in vitro. J. Pharmacol. Exp. Ther. 335, 553-561 (2010).

42. Kox, M. et al. Effects of the alpha7 nicotinic acetylcholine receptor agonist GTS-21 on the innate immune response in humans. Shock 36, 5-11 (2011)

43. Bruchfeld, A. et al. Whole blood cytokine attenuation by cholinergic agonists ex vivo and relationship to vagus nerve activity in rheumatoid arthritis. J. Intern. Med. 268, 94-101 (2010).

44. Godtfredsen, N. S., Lange, P., Prescott, E., Osler, M. \& Vestbo, J. Changes in smoking habits and risk of asthma: a longitudinal population based study. Eur. Respir. J. 18, 549-554 (2001).

45. Pietinalho, A., Pelkonen, A. \& Rytila, P. Linkage between smoking and asthma. Allergy 64, 1722-1727 (2009).

46. Siroux, V., Pin, I., Oryszczyn, M. P., Le Moual, N. \& Kauffmann, F Relationships of active smoking to asthma and asthma severity in the EGEA study. Epidemiological study on the Genetics and Environment of Asthma. Eur. Respir. J. 15, 470-477 (2000).

47. Marquette, C. H. et al. Long-term prognosis of near-fatal asthma. A 6-year follow-up study of 145 asthmatic patients who underwent mechanical ventilation for a near-fatal attack of asthma. Am. Rev. Respir. Dis. 146, 76-81 (1992).

48. Warren, C. P. Extrinsic allergic alveolitis: a disease commoner in non-smokers. Thorax 32, 567-569 (1977).

49. McSharry, C., Banham, S. W. \& Boyd, G. Effect of cigarette smoking on the antibody response to inhaled antigens and the prevalence of extrinsic allergic alveolitis among pigeon breeders. Clin. Allergy 15, 487-494 (1985). 
50. Anderson, K., Morrison, S. M., Bourke, S. \& Boyd, G. Effect of cigarette smoking on the specific antibody response in pigeon fanciers. Thorax 43, 798-800 (1988).

51. Carrillo, T. et al. Effect of cigarette smoking on the humoral immune response in pigeon fanciers. Allergy 46, 241-244 (1991).

52. Langhammer, A., Johnsen, R., Holmen, J., Gulsvik, A. \& Bjermer, L. Cigarette smoking gives more respiratory symptoms among women than among men. The Nord-Trondelag Health Study (HUNT). J. Epidemiol. Community Health 54, 917-922 (2000).

53. Linneberg, A. et al. Smoking and the development of allergic sensitization to aeroallergens in adults: a prospective population-based study. The Copenhagen Allergy Study. Allergy 56, 328-332 (2001).

54. Yu, H., Yang, Y. H., Rajaiah, R. \& Moudgil, K. D. Nicotine-induced differential modulation of autoimmune arthritis in the Lewis rat involves changes in interleukin-17 and anti-cyclic citrullinated peptide antibodies. Arthritis Rheum. 63, 981-991 (2011).

55. Shi, F. D. et al. Nicotinic attenuation of central nervous system inflammation and autoimmunity. J. Immunol. 182, 1730-1739 (2009).

56. Kearley, J. et al. Cigarette smoke silences innate lymphoid cell function and facilitates an exacerbated type I interleukin-33-dependent response to infection. Immunity 42, 566-579 (2015).

57. Barlow, J. L. et al. IL-33 is more potent than IL-25 in provoking IL-13producing nuocytes (type 2 innate lymphoid cells) and airway contraction. J. Allergy Clin. Immunol. 132, 933-941 (2013).

58. Dowling, O., Rochelson, B., Way, K., Al-Abed, Y. \& Metz, C. N. Nicotine inhibits cytokine production by placenta cells via NFkappaB: potential role in pregnancy-induced hypertension. Mol. Med. 13, 576-583 (2007).

59. Cesario, A., Russo, P., Nastrucci, C. \& Granone, P. Is alpha7-nAChR a possible target for lung cancer and malignant pleural mesothelioma treatment? Curr. Drug Targets 13, 688-694 (2012).

60. West, K. A. et al. Rapid Akt activation by nicotine and a tobacco carcinogen modulates the phenotype of normal human airway epithelial cells. J. Clin. Invest. 111, 81-90 (2003).

61. Dasgupta, P. et al. Nicotine inhibits apoptosis induced by chemotherapeutic drugs by up-regulating XIAP and survivin. Proc. Natl Acad. Sci. USA 103, 6332-6337 (2006)

62. Schaal, C. \& Chellappan, S. P. Nicotine-mediated cell proliferation and tumor progression in smoking-related cancers. Mol. Cancer Res. 12, 14-23 (2014).

63. Zheng, W. \& Flavell, R. A. The transcription factor GATA-3 is necessary and sufficient for Th2 cytokine gene expression in CD4 T cells. Cell 89, 587-596 (1997).

64. Ouyang, W. et al. Inhibition of Th1 development mediated by GATA-3 through an IL-4-independent mechanism. Immunity 9, 745-755 (1998).

65. Ansel, K. M., Djuretic, I., Tanasa, B. \& Rao, A. Regulation of Th2 differentiation and Il4 locus accessibility. Annu. Rev. Immunol. 24, 607-656 (2006).

66. Liang, H. E. et al. Divergent expression patterns of IL-4 and IL-13 define unique functions in allergic immunity. Nat. Immunol. 13, 58-66 (2012).
67. Kerzerho, J. et al. Programmed cell death ligand 2 regulates TH9 differentiation and induction of chronic airway hyperreactivity. J. Allergy Clin. Immunol. 131, 1048-1057 (2013).

68. Maazi, H. et al. Lack of PD-L1 expression by iNKT cells improves the course of influenza A infection. PloS ONE 8, e59599 (2013).

69. Aizawa, K. et al. Development of ferret as a human lung cancer model by injecting 4-(Nmethyl-N-nitrosamino)-1-(3-pyridyl)-1-butanone (NNK). Lung Cancer 82, 390-396 (2013).

70. Suzuki, Y. et al. Lack of autophagy induces steroid-resistant airway inflammation. J. Allergy Clin. Immunol. 137, 1382-1389.e1389 (2016).

\section{Acknowledgements}

Studies described in this article were financially supported by National Institutes of Health Public Health Service Grant R01AI066020, R01ES025786, R01ES021801 (OA) and SC-CTSI Grant UL1TR000130 (OA and LC).

\section{Author contributions}

Y.S. and L.G.-T. performed and analysed experiments. N.P. and H.M. performed RNA seq and NanoString analysis. I.S. provided materials and procured mice. Y.S., L.G.-T. and J.L.A. wrote the manuscript. L.C. provided nicotine agonist and information regarding nicotine agonists necessary for the study; L.C. also proof read the manuscript. O.A. conceptualized and designed the experiments, supervised the work and proof read the manuscript.

\section{Additional information}

Supplementary Information accompanies this paper at http://www.nature.com/ naturecommunications

Competing financial interests: The authors declare no competing financial interests.

Reprints and permission information is available online at http://npg.nature.com/ reprintsandpermissions/

How to cite this article: Galle-Treger, L. et al. Nicotinic acetylcholine receptor agonist attenuates ILC2-Dependent airway hyperreactivity. Nat. Commun. 7, 13202 doi: $10.1038 /$ ncomms13202 (2016).

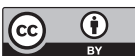

This work is licensed under a Creative Commons Attribution 4.0 International License. The images or other third party material in this article are included in the article's Creative Commons license, unless indicated otherwise in the credit line; if the material is not included under the Creative Commons license, users will need to obtain permission from the license holder to reproduce the material. To view a copy of this license, visit http://creativecommons.org/licenses/by/4.0/

(C) The Author(s) 2016 